2 Title: $\mathrm{Pl}(4,5) \mathrm{P}_{2}$ Binding Sites in the Ebola Virus Matrix Protein Modulate

3 Assembly and Budding

4

5 Authors: Kristen A. Johnson ${ }^{1,7}$, Melissa R. Budicini ${ }^{2}$, Sarah Urata $^{3}$, Nisha

6 Bhattarai ${ }^{4}$, Bernard S. Gerstman ${ }^{4,5}$, Prem P. Chapagain ${ }^{4,5}$, Sheng Li ${ }^{3}$ and Robert

$7 \quad$ V. Stahelin ${ }^{1,6, *}$

9 Author Affiliations: ${ }^{1}$ Department of Chemistry and Biochemistry, University of Notre

10 Dame, Notre Dame, IN 46556, ${ }^{2}$ Department of Biology, University of Notre Dame, Notre

11 Dame, IN 46556, ${ }^{3}$ Department of Medicine, University of California, San Diego, La Jolla,

12 CA 92093-0652, ${ }^{4}$ Department of Physics and ${ }^{5}$ Biomolecular Sciences Institute, Florida

13 International University, Miami, FL 33199, ${ }^{6}$ Department of Medicinal Chemistry and

14 Molecular Pharmacology Purdue University, West Lafayette, IN 47907.

$15{ }^{7}$ Current address: Department of Molecular Genetics, University of Texas Southwestern

16 Medical Center, Dallas, TX 75390.

17 *To whom correspondence should be addressed: rstaheli@purdue.edu

\title{
18 Abstract:
}

19 Ebola virus (EBOV) causes sever hemorrhagic fever in humans, can cause death

20 in a large percentage of those infected, and still lacks FDA approved treatment

21 options. In this study, we investigated how the essential EBOV protein, VP40,

22 forms stable oligomers to mediate budding and assembly from the host cell

23 plasma membrane. An array of in vitro and cellular assays identified and

24 characterized two lysine rich regions that bind to $\mathrm{PI}(4,5) \mathrm{P}_{2}$ and serve distinct 
25 functions through the lipid binding and assembly of the viral matrix layer. We

26 found that when VP40 binds $\mathrm{PI}(4,5) \mathrm{P}_{2}$, VP40 oligomers become extremely stable

27 and long lived. Together, this work characterizes the molecular basis of $\mathrm{PI}(4,5) \mathrm{P}_{2}$

28 binding by VP40, which stabilizes formation of VP40 oligomers necessary for

29 viral assembly and budding. Quercetin, a natural product that lowers $\mathrm{PI}(4,5) \mathrm{P}_{2}$ in

30 the plasma membrane, inhibited budding of VP40 VLPs and may inform future

31 treatment strategies against EBOV.

\section{Introduction:}

34 Ebola virus (EBOV) is a lipid-enveloped pleomorphic virus that can infect a wide

35 variety of human cells causing significant disease $e^{1,2}$. To date there are no FDA

36 approved drugs or vaccines for EBOV treatment and there is still a great deal of

37 need in understanding how to therapeutically target the five different types of

38 ebolaviruses. The EBOV glycoprotein (GP), which studs the exterior of the virus

39 particle during the infection scheme, has been the major target of vaccine

40 development and antibody based therapies. Indeed, a vaccine using EBOV GP

41 pseudotyped into vesicular stomatitis virus is being implemented in the current

42 EBOV outbreak in the Democratic Republic of Congo ${ }^{3}$. While many of the

43 emerging treatments aimed at the GP show great promise, the GP has been

44 shown to mutate significantly during the course of the recent outbreak ${ }^{4-6}$ and a

45 watch list of potential GP antibody escape mutants has been generated ${ }^{7}$. Thus,

46 other targets in the viral life cycle are being explored such as host-viral

47 interactions ${ }^{8}$ and the matrix protein VP40 8 -10. 
VP40 is the matrix protein of EBOV and required for viral assembly, budding

49 and viral spread ${ }^{11-16}$. As the most abundantly expressed EBOV protein, VP40

50 achieves several distinct and essential tasks to ensure viral success. VP40 is a

51 transformer protein with several structures known, including a monomer ${ }^{17}$, dimer,

52 hexamer and octamer ${ }^{18}$. Initially, VP40 enters the nucleus of the host cell post

53 viral entry ${ }^{19}$, likely binds RNA as an octamer and regulates viral genome

54 replication ${ }^{18,20}$. Subsequently, VP40 dimers interact with host lipids such as

55 phosphatidylserine (PS) $)^{21,22}$ to localize to the plasma membrane $(P M)^{18,22}$ and

56 form stable hexamers ${ }^{18,21,23-25}$. Phosphatidylinositol 4,5-bisphosphate $\left(\mathrm{PI}(4,5) \mathrm{P}_{2}\right)$

57 at the host PM inner leaflet is required for large VP40 oligomers to form ${ }^{26}$ from $^{2}$

58 PS induced VP40 hexamers ${ }^{18,21-25}$.

59 Despite an important role for $\mathrm{PS}$ and $\mathrm{PI}(4,5) \mathrm{P}_{2}$ in the EBOV life cycle and

60 budding from the host cell PM, the molecular basis and structural consequences

61 of VP40-lipid interactions are mostly unknown. Notably, the VP40 dimer is

62 necessary for PM localization whereas the monomer is not sufficient for

63 trafficking to the $P M^{18,22}$. Dimer interactions with $\mathrm{PM}$ lipids are purported to

64 induce structural changes to form VP40 hexamers ${ }^{18,21,23-25,27}$ and larger

65 oligomers forming the viral matrix layer ${ }^{23,26}$. Notably, the VP40 octamer has been

66 shown to play an important role in the regulation of viral $\operatorname{transcription}^{18}$ and has

67 not been detected at the PM nor in virions or VLPs. Additionally, the VP40

68 octamer was found to have a significant reduction in PS affinity compared to the

69 VP40 dimer ${ }^{22}$. 
70 Previously, we found that VP40 requires $\mathrm{PI}(4,5) \mathrm{P}_{2}$ at the plasma

71 membrane for the formation of large self-oligomers, VLP formation, and VLP

72 budding ${ }^{26}$. A recent molecular dynamics model found VP40 hexamers cluster

$73 \mathrm{PI}(4,5) \mathrm{P}_{2}$ but not PS at the PM through C-terminal lysine residues ${ }^{28}$. In this study,

74 we investigate the VP40 lipid binding properties at the PM and the structural

75 consequences of interactions with $\mathrm{PS}$ and $\mathrm{PI}(4,5) \mathrm{P}_{2}$. Results demonstrate that

$76 \mathrm{PI}(4,5) \mathrm{P}_{2}$ provides significant structural stability to VP40 hexamers that form

77 following interactions with $\mathrm{PS}$. $\mathrm{PI}(4,5) \mathrm{P}_{2}$ provides extensive stability to the viral

78 matrix layer through two distinct $\mathrm{PI}(4,5) \mathrm{P}_{2}$ binding sites, loss of which leads to

79 rapid dissociation of VP40 oligomers and inhibition of viral budding. Further, a

80 natural product Quercetin, which reduces plasma membrane $\mathrm{PI}(4,5) \mathrm{P}_{2}$

81 significantly inhibited VLP formation from the PM. Taken together, these new

82 findings account for the molecular basis of $\mathrm{Pl}(4,5) \mathrm{P}_{2}$ recognition by VP40,

$83 \mathrm{PI}(4,5) \mathrm{P}_{2}$ induced structural stability and assembly of VP40, and open up a

84 potentially new avenue for EBOV treatment.

\section{Results:}

86 Hypothesized $\mathrm{PI}(4,5) \mathrm{P}_{2}$ binding site is conserved among ebolaviruses:

87 VP40 from Zaire, Sudan, Tai Forest, Bundibugyo, and Reston ebolaviruses were

88 aligned using uniprot to reveal sequence conservation in residues hypothesized

89 to bind to $\mathrm{PI}(4,5) \mathrm{P}_{2}$. Lys ${ }^{224}, \mathrm{Lys}^{225}$, Lys ${ }^{274}$, and Lys ${ }^{279}$ are completely conserved

90 while Lys ${ }^{221}$ has an Arg in the Tai Forest strain (Figure 1A). Lys ${ }^{225}$ is Arg in the

91 Bundibugyo strain while residue 270 is Arg or Lys (Figure 1A). Both Arg and Lys 
92 are commonly found in $\mathrm{PI}(4,5) \mathrm{P}_{2}$ binding sites ${ }^{29}$. To test if these VP40 residues

93 are involved in $\mathrm{PI}(4,5) \mathrm{P}_{2}$ binding, alanine scanning mutagenesis was used for $\mathrm{C}$ -

\begin{tabular}{|c|c|c|c|c|}
\hline Query & Strain & AA & Sequence & AA Sequence \\
\hline Q05128 & VP40_EBOZM & 220 & NKSGKKGN & 269 HKLTGKKVTSKNGQPI \\
\hline Q5XX06 & VP40_Sudan & 220 & GKTGKKGH & 269 HKLTGKKMSQKNGQPI \\
\hline $\mathrm{B} 8 \mathrm{X} \mathrm{CN} 8$ & VP40_Tai Forest & 220 & GRAGKKGS & 269 HRLTGKKTTTKNGQPI \\
\hline B8XCN9 & VP40_Bundibugyo & 220 & GKTGKRGS & 269 HRLTGKKITTKNGQPI \\
\hline L7REV2 & VP40_Reston & 220 & GKIGKKGH & 269 QRLTGKKPQPKNGQPI \\
\hline
\end{tabular}

B.
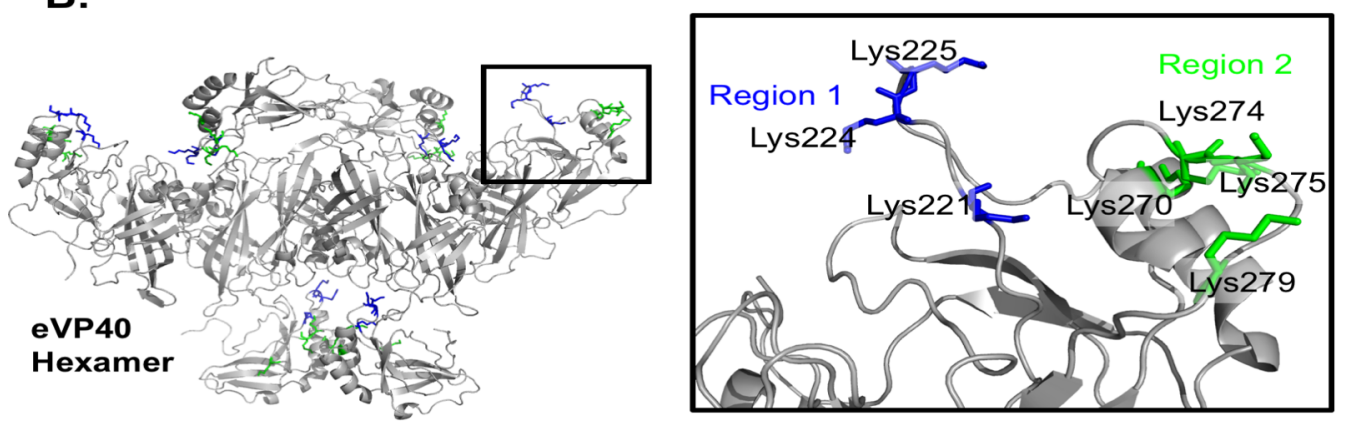

Figure 1

Figure 1: Ebola VP40 sequence and structure revels two $\mathrm{PI}(4,5) \mathrm{P}_{2}$ binding sites. A. Consensus sequence of Ebola VP40 strains several lysine residues in the 220 and 270 regions of the protein are positioned at the surface of the C-terminal domain. Sequence alignment was performed with uniprot alignment tools. B. Zaire VP40 hexamer structure (4ldd with modeled Cterminal domains) with Region 1 (220 lysine residues) colored in blue and Region 2 (270 lysine residues) colored in green.

94 terminal domain regions with high conservation across eboaviruses. These

95 mutations include Lys ${ }^{104}$, Lys $^{221}$, Lys $^{224}$, Lys $^{225}$, Lys $^{236}$, His $^{269}$, Lys $^{270}$, Lys $^{274}$,

96 Lys $^{275}$, Lys $^{279}$, Lys $^{291}$. When mapped onto the VP40 hexamer model, the 220's

97 lysine residues (blue) and the 270's lysine residues (green) formed distinct,

98 adjacent domains (Figure 1B).

99 VP40 binds to $\mathrm{PI}(4,5) \mathrm{P}_{2}$ containing membranes with high selectivity among

100 phosphoinositides: 
VP40 binding to phosphoinositides (PI(3)P, PI(4)P, PI(5)P, PI(3,4) $\mathrm{P}_{2}$,

$102 \mathrm{PI}(3,5) \mathrm{P}_{2}, \mathrm{PI}(4,5) \mathrm{P}_{2}$, and $\left.\mathrm{PI}(3,4,5) \mathrm{P}_{3}\right)$ was quantified using a raffinose

103 pentahydrate loaded large unilamellar liposome (LUV) pelleting assay ${ }^{30}$. The

104 liposome composition included 1,2-dipalmitoyl-sn-glycero-3-phosphocholine

105 (DPPC) and cholesterol 1:1 with 5\% phosphoinositide and 2\% dansylPE.

106 Previously, we did not observe binding to $\mathrm{PI}(4,5) \mathrm{P}_{2}$ containing multilamellar

107 vesicles (MLVs) without 40\% POPS added to the PIP ${ }^{31}$. However, we found that

108 VP40 binds well to $\mathrm{PI}(4,5) \mathrm{P}_{2}$ in the LUV system, which better recapitulates

109 cellular membrane bilayers. The binding to $\mathrm{PI}(4,5) \mathrm{P}_{2}$ was especially notable in

110 the presence of ordered lipids DPPC and cholesterol when compared to POPC

111 and DOPE (See Figure S1A and S1B). To ensure LUV formation, dynamic light

112 scattering was used to measure the diameter of extruded liposomes (data not

113 shown). Pelleting efficiency was quantified by including $2 \%$ dansylPE in the

114 liposomes, the percent fluorescence in the supernatant (SN) and pellet $(\mathrm{P})$

115 fractions were quantified (Ex 355, Em 510). Pelleting efficiency for this liposome

116 composition was approximately 100\% (Figure S1C).

117 In this LUV system, VP40 displayed significant binding to PI(4)P,

$118 \mathrm{PI}(3,4) \mathrm{P}_{2}$, and $\mathrm{PI}(4,5) \mathrm{P}_{2}$ with the most significant binding to $\mathrm{PI}(4,5) \mathrm{P}_{2}$.

119 Interestingly, binding to $\mathrm{PI}(3,5) \mathrm{P}_{2}$ was not observed (Figure 2A and 2B). VP40

120 selectivity for $\mathrm{PI}(4,5) \mathrm{P}_{2}$ over the other 6 phosphoinositide species indicated there

121 was likely a lipid binding site driving the selectivity and additionally, previous

122 studies ruled out nonspecific electrostatics as a driving force of EBOV VP40

123 assembly ${ }^{21,26}$. 
A.

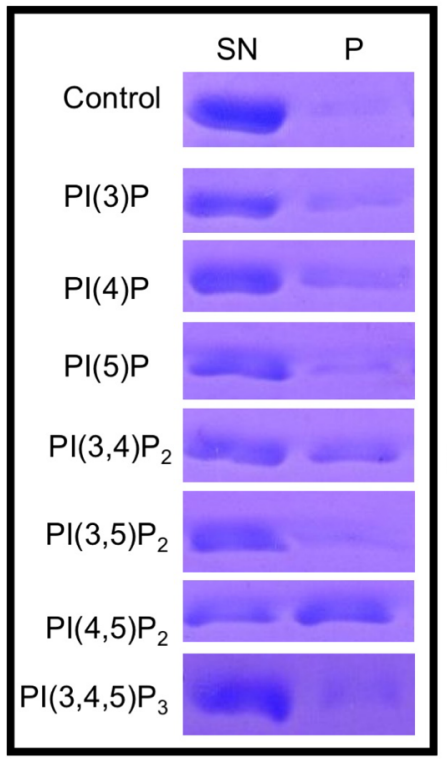

B.

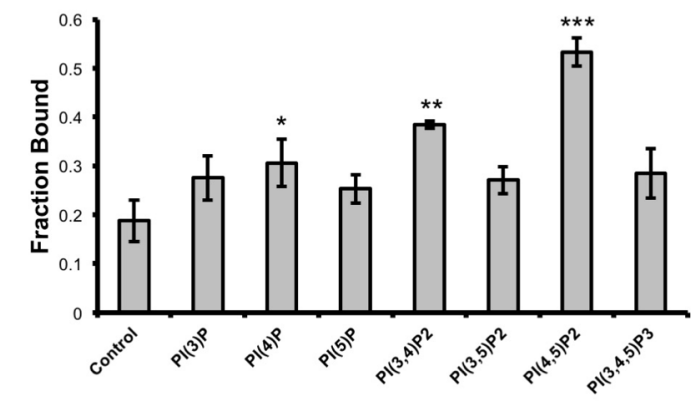

C.

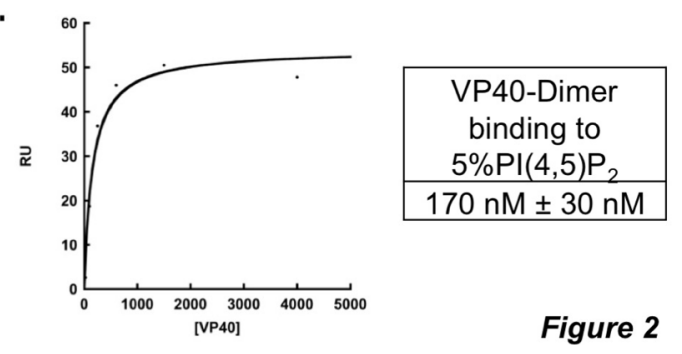

Figure 2: VP40 binds to $\mathrm{PI}(4,5) \mathrm{P}_{2}$ with selectivity and nanomolar affinity. A. Representative SDS PAGE bands from the large unilamellar (LUV) pelleting assay with DPPC:Cholesterol without (control) or with $5 \%$ of one of the seven phosphatidylinositol phosphate species. B. Quantified VP40 fraction bound to liposomes (protein found in pellet fraction). The average value is plotted and shown \pm the SEM. C. Representative surface plasmon resonance curve of VP40 binding to liposomes containing $5 \% \mathrm{PI}(4,5) \mathrm{P}_{2}$ gave an average binding affinity of $170 \mathrm{nM}$ with a standard error of the mean of $26 \mathrm{nM}$. ${ }^{*} \mathrm{P}<0.05,{ }^{* *} \mathrm{P}<0.001$, ${ }^{* * *} \mathrm{P}<0.00001$

Surface plasmon resonance (SPR) was used to determine the affinity of

125 VP40 for $\mathrm{PI}(4,5) \mathrm{P}_{2}$ as previously described ${ }^{32}$. Using this technique, we found that

126 the VP40 dimer binds to $\mathrm{PI}(4,5) \mathrm{P}_{2}$ containing LUVs with an apparent affinity of

$127170 \mathrm{nM}$ (Figure 2). This binding affinity is also slightly stronger than the

128 interaction between VP40 and POPS, also determined with SPR ${ }^{21}$.

129 VP40 binds to $\mathrm{PI}(\mathbf{4 , 5}) \mathrm{P}_{\mathbf{2}}$ through lysine residues in CTD Region 1 and

130 Region 2:

131 To determine which VP40 residues are responsible for $\mathrm{PI}(4,5) \mathrm{P}_{2}$

132 interactions, lipid binding of VP40 lysine mutants was tested in vitro using a the

133 LUV assay detailed above. Binding to liposomes containing $5 \% \mathrm{PI}(4,5) \mathrm{P}_{2}$ was 
134 measured for the dimer of VP40-WT and select lysine mutants. VP40-K104A and

$135 \mathrm{~K} 236 \mathrm{~A}$ did not have a significant difference in binding to $\mathrm{PI}(4,5) \mathrm{P}_{2}$ LUVs

136 compared to the WT protein (Figure 3). In contrast, K221A, K224A, K225A,

$137 \mathrm{~K} 270 \mathrm{~A}, \mathrm{~K} 274 \mathrm{~A}, \mathrm{~K} 275 \mathrm{~A}$, and K279A had significant decreases in binding to LUVs 138 containing $\mathrm{Pl}(4,5) \mathrm{P}_{2}$ compared to WT (Figure 3).

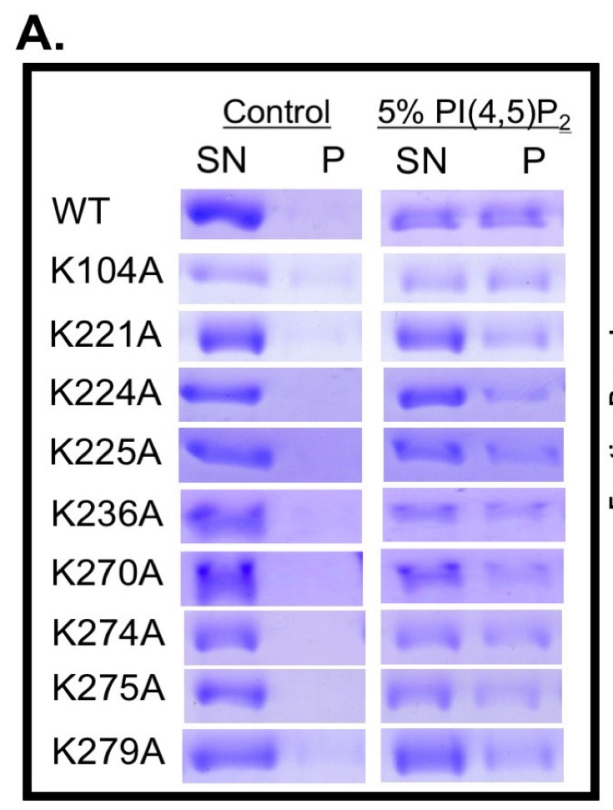

\section{B.}

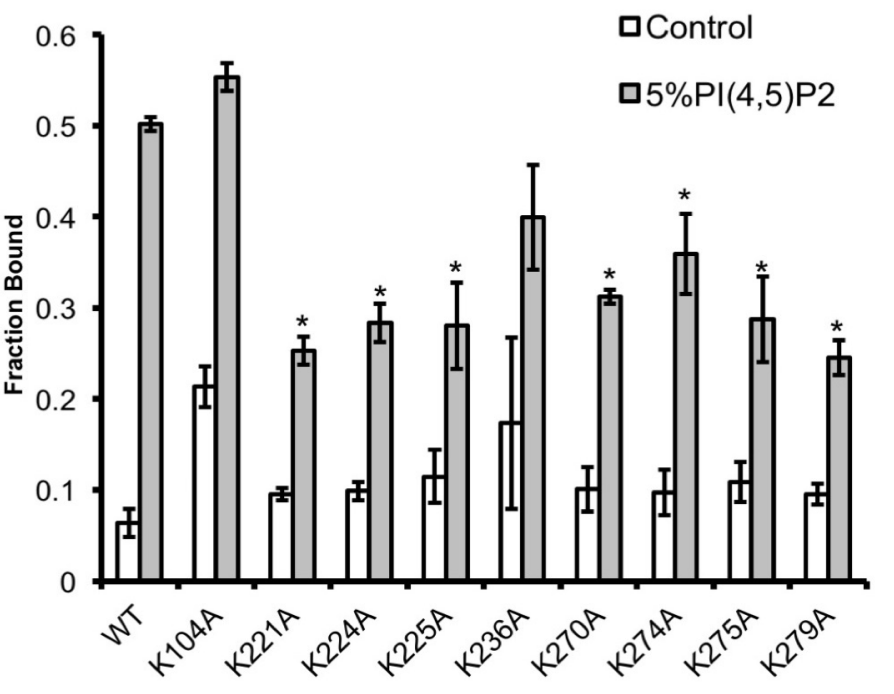

Figure 3

Figure 3: VP40 C-terminal domain lysine residues are important for $\mathrm{PI}(4,5) \mathrm{P}_{2}$ binding. A. Representative SDS PAGE of the LUV pelleting assay to measure VP40 binding to control (DCCP and Cholesterol) or $5 \% \mathrm{PI}(4,5) \mathrm{P}_{2}$. B. Average fraction bound to LUVs shown \pm the SEM, * $P<0.05$.

$139 \mathrm{PI}(4,5) \mathrm{P}_{2}$ binding residues are critical for the VP40 budding phenotype in

140 live cells:

In COS-7 cells, WT VP40-EGFP produces an abundance of VLPs at the

142 PM 12-14 hours post transfection (Figure 4A) ${ }^{26}$. To screen the phenotype of

143 VP40 C-terminal mutants, each EGFP-construct was expressed in COS-7 cells.

144 The percentage of cells producing VLPs were compared to WT-VP40 (Figure

145 4B), representative images of each are shown in Figure 4A. Mutations that 

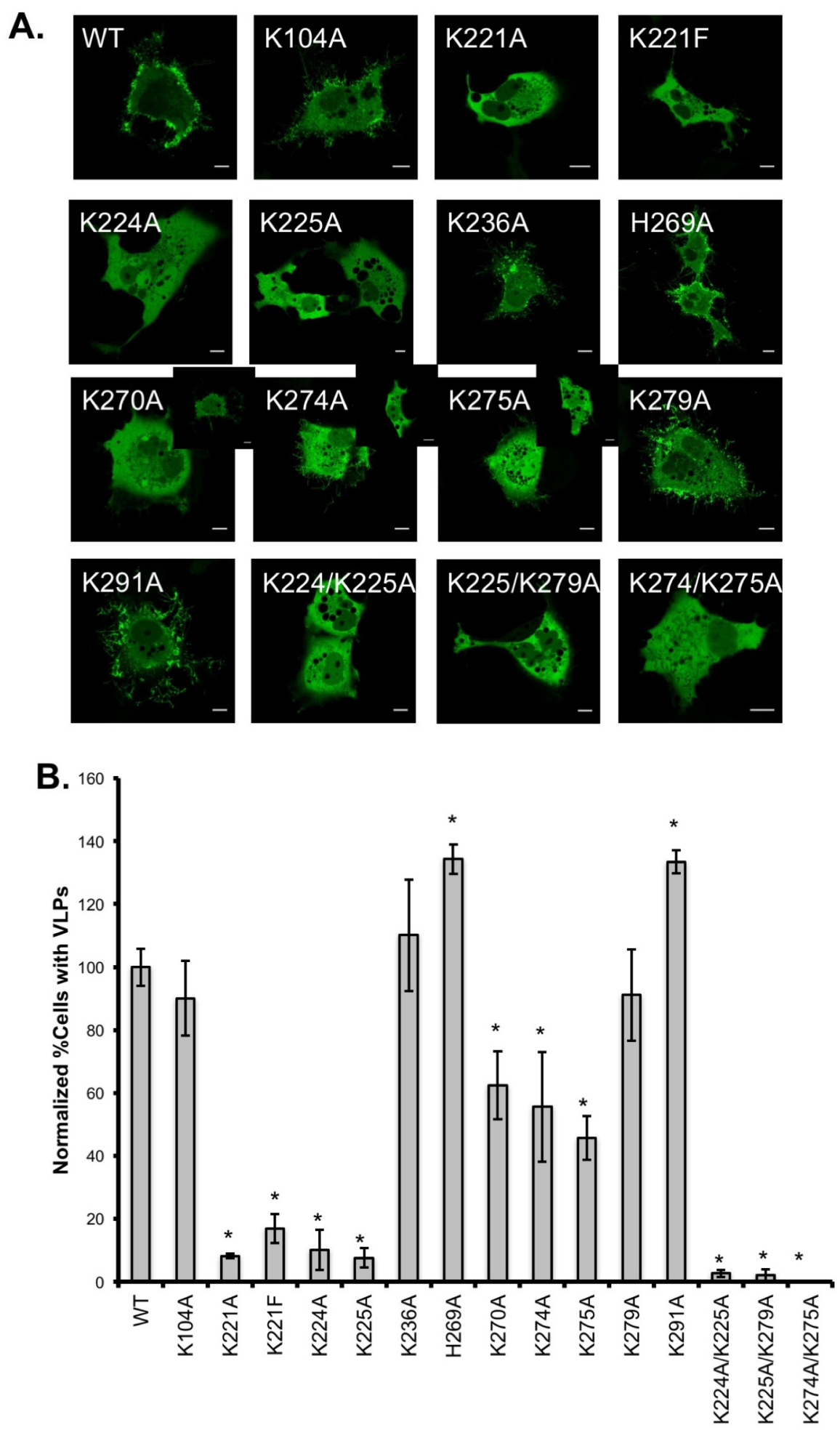

Figure 4: VP40 mutant phenotype in live cells. A. Representative images of WTVP40-EGFP and VP40 mutants 14 hours post transfection in COS-7 cells. Scale bars are $10 \mu \mathrm{m}$. B. Percentage of the cell population expressing VLPs 14 hours post transfection, normalized to WT. Average values are shown \pm the SEM, * $\mathrm{P}<0.05$.

146 showed two phenotypes have a second, smaller representative image to show 
147 the minor phenotype. K221A and K225A had the most dramatic reduction in VLP

148 production with fewer than $10 \%$ of cells producing VLPs. Less than $20 \%$ of cells

149 expressing K221F and K224A showed VLPs and about $50 \%$ of cells expressing

$150 \mathrm{~K} 270 \mathrm{~A}, \mathrm{~K} 274 \mathrm{~A}$, or K275A had detectable VLPs. Some lysine to alanine

151 mutations (K104A, K236A, K279A, K291A) had a phenotype similar to WT.

152 Scanning electron microscopy (SEM) was used to achieve high resolution

153 of the PM of VP40 transfected cells. VP40-EGFP-WT, K221A, K104A, K224A,

154 K274A, K279A, and non-transfected COS-7 cells were prepared over two

155 independent experiments (Figure 5B and 5C). COS-7 cells have naturally

156 occurring filopodia structures observed in SEM (see Figure S2). Because of

157 this, filopodia structures were measured in FIJI to determine the average control

158 and VP40 transfected cell filopodia length. We found that control cells have an

159 average of $1 \mu \mathrm{m}$ filopodia while VP40 WT transfected cells have closer to $2 \mu \mathrm{m}$

160 VLPs (Figure 5). The VLP length was also compared to EGFP-VP40 from

161 confocal imaging (Figure 5). In consonance with the confocal imaging, K104A

162 and K279A had similar VLP structures to WT-VP40. K274A had similar length

163 but less VLP density and K221A and K224A had filopodia structures more similar

164 to the non-transfected control than WT-VP40 (See Figure 5).

$165 \mathrm{PI}(4,5) \mathrm{P}_{2}$ binding resides in Region 1 are critical for VP40 oligomers larger

166 than a hexamer:

167 A VP40 oligomerization assay in live cells ${ }^{21,23-26,33}$ was used to compare

168 oligomerization abilities of mutants deficient in $\mathrm{PI}(4,5) \mathrm{P}_{2}$ binding compared to 
169 WT. Previously, when $\mathrm{PI}(4,5) \mathrm{P}_{2}$ was depleted from the $\mathrm{PM}$ of live cells with a

170 Myc5-PtaselV construct, VP40 large oligomer formation was significantly
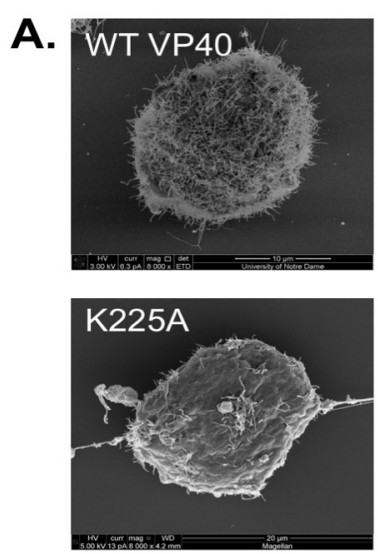

B.
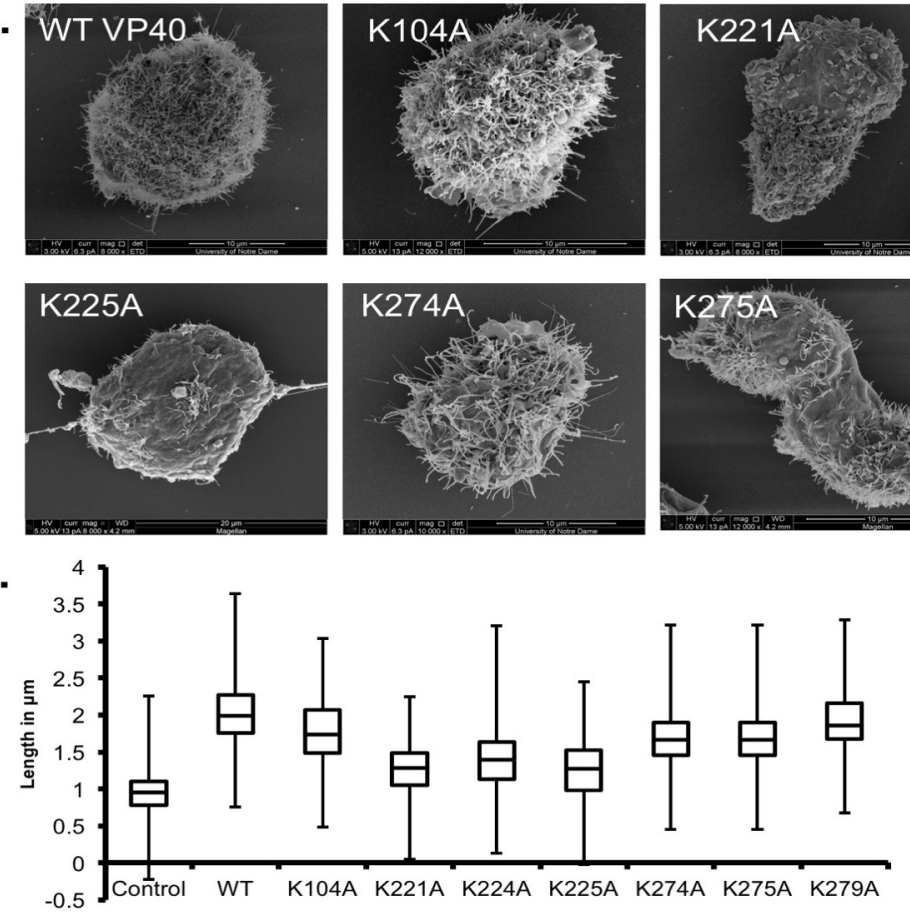
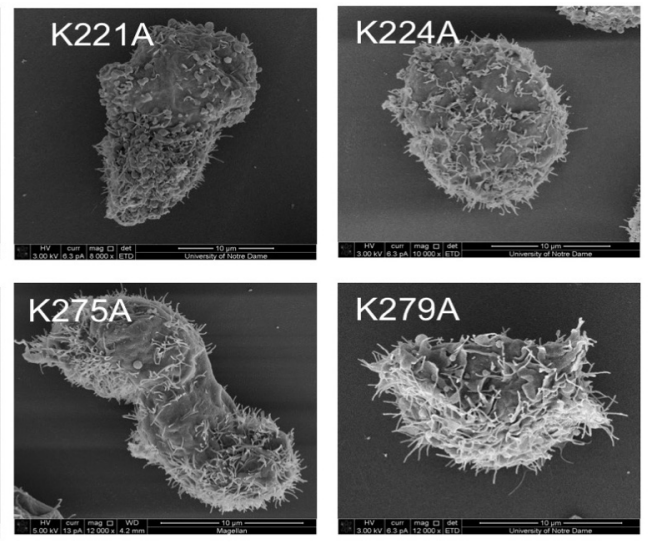

Figure 5

Figure 5: Scanning electron microscopy of VP40 expressing cells to assess virus like particle production. A. Representative SEM images of COS-7 cells expressing WT-VP40-EGFP and mutant-VP40-EGFP constructs. B. Box and whisker plot shows VLP lengths for WT and mutant VP40s. Values represent the average VLP length where 20 VLPs measured per image per mutant, data represents two independent experiments.

171 reduced $^{31}$. Adu-Gyamfi et al. found VP40 hexamer formation was dependent on

172 plasma membrane $\mathrm{PS}^{21}$. Together, this suggests different roles for PS and

$173 \mathrm{PI}(4,5) \mathrm{P}_{2}$ in the structure and assembly of the viral matrix layer.

We assessed the oligomer formation of VP40 mutants and found that

$175 \mathrm{~K} 221 \mathrm{~A}, \mathrm{~K} 224 \mathrm{~A}$, and K225A had a dramatic and significant reduction in oligomers

176 larger than a hexamer (Figure 6). For these mutants, there was also a significant

177 increase in the hexamer population compared to WT VP40. K270A, K274A, and 
179 some large oligomers were detected (Figure 6). This data recapitulates what

180 was observed previously when $\mathrm{PI}(4,5) \mathrm{P}_{2}$ was constitutively depleted with

181 Myc5'Ptase IV constructs ${ }^{31}$ but suggests the two cationic regions may contribute

182 differently to the oligomerization and stabilization process.

A.
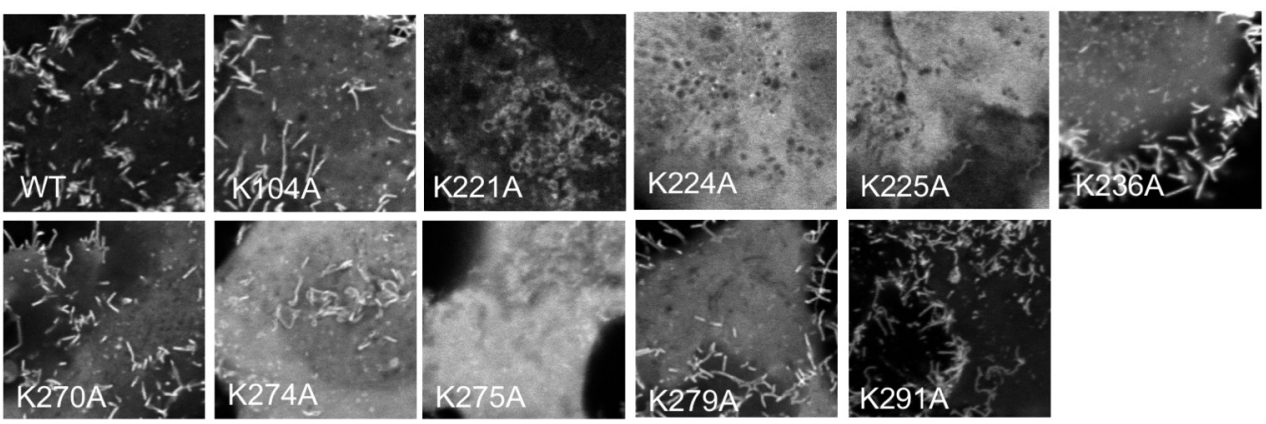

B.

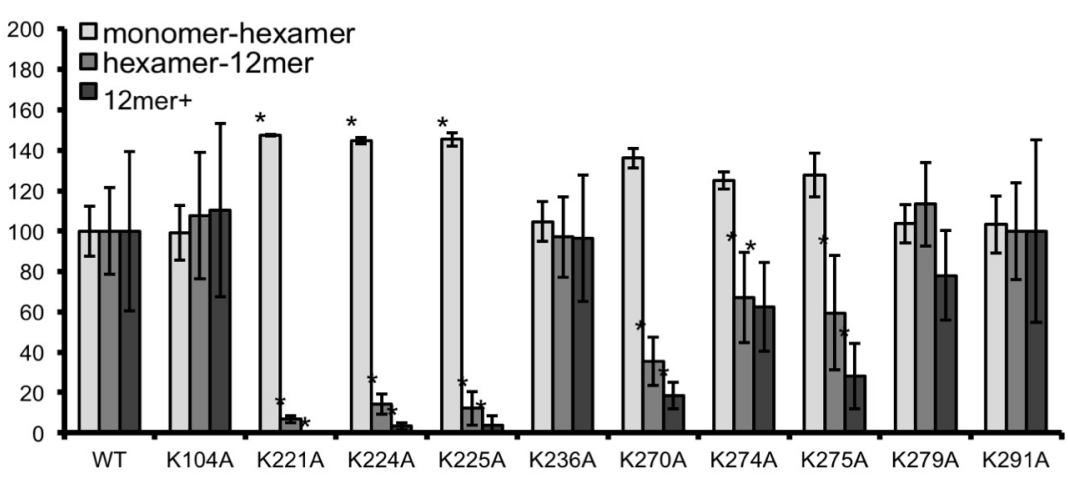

Figure 6

Figure 6: Number and Brightness analysis to determine VP40 oligomerization in live cells. A. Representative image frame from the 100 images acquired per for the imaging experiment. All are imaged at the same zoom (16.4). B. Normalized VP40 oligomer formation. The average WT-VP40-EGFP population was normalized to $100 \%$ for monomer-hexamer, hexamer-12mer, and 12mer+. WT and mutant VP40EGFP oligomer population values are shown \pm the SEM, ${ }^{*} \mathrm{P}<0.05$.

$\mathrm{PI}(4,5) \mathrm{P}_{2}$ binding residues are critical for efficient virus like particle

184 budding:

Next, VLPs and cell lysates of VP40 transfected COS-7 cells were

186 collected 20-24 hours post transfection (see Methods for more details). The

187 budding efficiency of WT VP40 and select mutants was determined with western

188 blot analysis. Surprisingly, K221A has a similar budding efficiency to WT while 
189 K221A expression levels were consistently lower than WT. K221A VLPs were

190 not commonly observed in confocal microscopy (Figure 5B) or scanning electron

191 microscopy (Figure 6B) and oligomer formation was significantly reduced

192 (Figure 7B).

193 K104A exhibited a decrease in VLP formation. This mutant is interesting

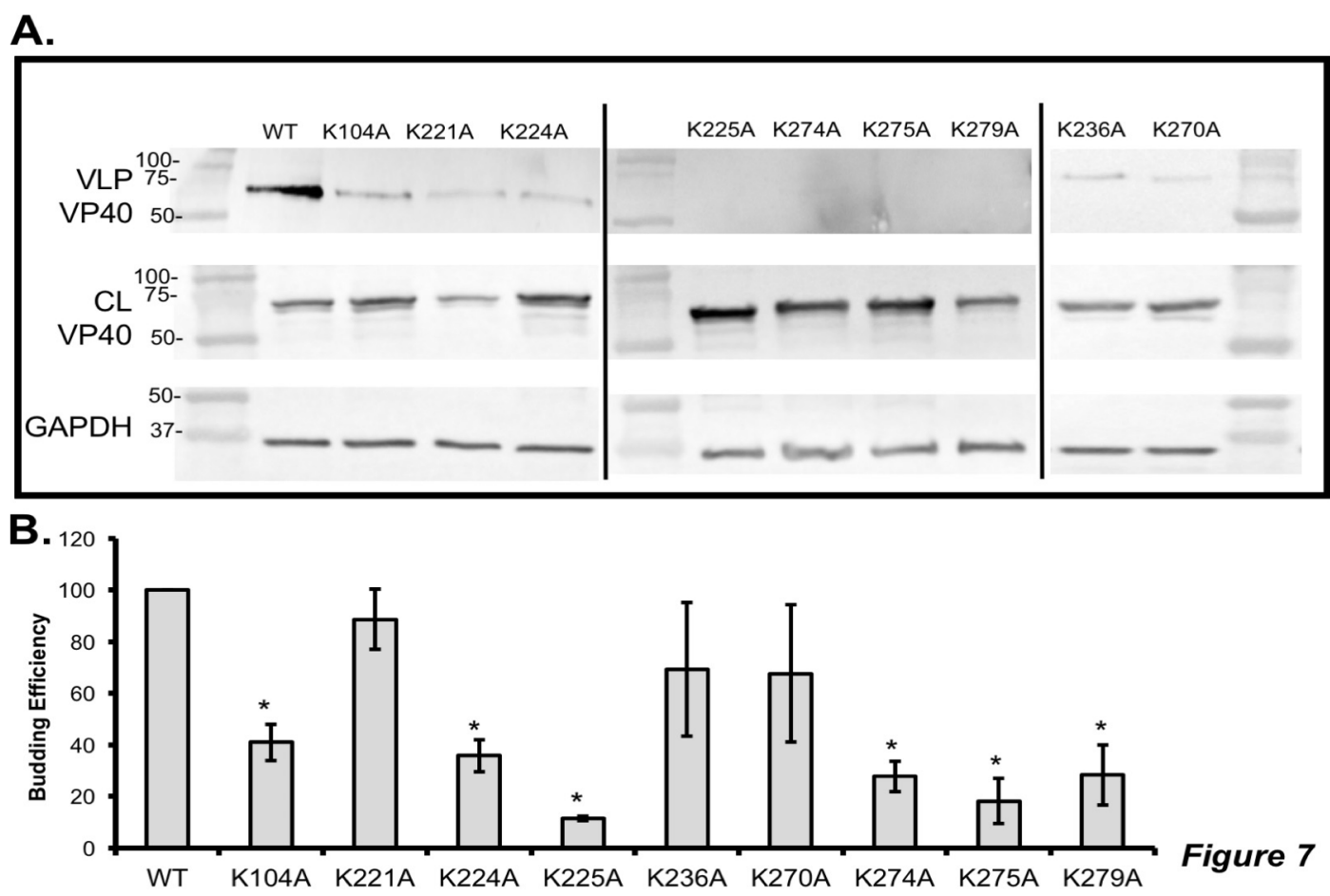

Figure 7: VLP budding efficiency of WT and mutant VP40. A. Western blots of WT and mutant VP40-EGFP show VP40 in the VLP and the cell lysate (CL) fractions. Housekeeping protein, GAPDH (CL), was used as a loading control for these experiments. Molecular weight markers are shown for all blots to verify band size for VP40-EGFP and GAPDH. B. Quantified budding efficiency normalized to VP40-WT. Average values are shown \pm the SEM, ${ }^{*} P<0.05$.

194 as it is in the $\mathrm{N}$-terminal domain but folds against the C-terminal domain adjacent

195 to aspartic acid residue 193 in the dimer. This suggests its interaction with lipid

196 may not be a major contributor to $\mathrm{PI}(4,5) \mathrm{P}_{2}$ dependent assembly but a role in

197 interdomain interactions cannot be discounted at this time. K279A also showed

198 a significant decrease in budding which is consistent with the reduction in binding 
199 to $\mathrm{PI}(4,5) \mathrm{P}_{2}$ containing membranes in vitro (Figure 3). This result was partially

200 unexpected as VLPs are observed in confocal microscopy (Figure 4B) and SEM

201 (Figure 5B). However, this mutation had a significant decrease in $\mathrm{PI}(4,5) \mathrm{P}_{2}$

202 binding in the LUV pelleting assay (Figure 3B) and a small decrease in

203 oligomerization (Figure 6). Because Lys ${ }^{279}$ is important for lipid binding in vitro,

204 we hypothesize this indicates that even a small decrease in $\mathrm{PI}(4,5) \mathrm{P}_{2}$ binding

205 and self-oligomerization may have dramatic effects on budding efficiency.

206 Hydrogen deuterium exchange mass spectrometry reveals VP40 oligomers

207 become extremely stable following $\mathrm{PI}(4,5) \mathrm{P}_{2}$ binding:

208 Hydrogen deuterium exchange mass spectrometry (HDX MS) was used to

209 better understand structural changes in VP40 upon binding to PS and $\mathrm{PI}(4,5) \mathrm{P}_{2}$

210 containing liposomes. Complete coverage analysis of the VP40 protein was

211 achieved with many overlapping peptides (Figure S3). To ensure a

212 homogeneous sample, elevated levels of $\mathrm{PI}(4,5) \mathrm{P}_{2}$ or PS were used (Figure S4).

213 Samples were incubated for 10,100, 1000, 10,000 and 100,000 seconds (s).

214 Changes in deuterium incorporation during these time points are shown in

215 Figures S5-10. Supplemental figures 4, 6, and 8 show ribbon maps with the

216 level of deuterium incorporation mapped to the protein sequence. Supplemental

217 figures 5, 7, and 9 show the influence of $\mathrm{PC}, \mathrm{PS}$, and $\mathrm{PI}(4,5) \mathrm{P}_{2}$, respectively.

218 Figure S10 shows the HDX data mapped onto the dimer structure and Figure

219 S11 shows the influence of each lipid on the VP40 dimer structure at 10,000 s.

220 The deuterium incorporations for each time point and lipid were also mapped to

221 the VP40 hexamer structure, the relevant structure for VP40 matrix assembly ${ }^{18}$. 
Figure 8A shows the percent deuterium incorporation mapped to the

223 hexamer structure while Figure 8B shows the influence of $\mathrm{PC}, \mathrm{PS}$, and $\mathrm{PI}(4,5) \mathrm{P}_{2}$

224 on the hexamer structure. In the presence of PC, VP40 deuterium exchange is

225 slightly increased compared to without lipid as shown by the light red regions in

226 the NTD. The VP40 structural changes observed through decreased deuterium

227 incorporation after binding $\mathrm{PI}(4,5) \mathrm{P}_{2}$ were very stable with less than $20 \%$ change

228 in deuterium incorporation between the $10 \mathrm{~s}$ and $100,000 \mathrm{~s}$ time points. The

229 majority of decreases in deuterium incorporation were lost in the PS samples

230 after the 10,000s time point (Figure 8C-D). Again, this data strongly supports a

231 model where PS is important for initial membrane association and VP40

232 conformational change whereas $\mathrm{PI}(4,5) \mathrm{P}_{2}$ plays a more predominant role in

233 stabilizing the VP40 oligomers that form.

234 Deuterium incorporation was reduced at all time points in the regions of

235 the proposed $\mathrm{PI}(4,5) \mathrm{P}_{2}$ interacting residues. Interestingly, regions containing lys

236 residues in region $220\left(\right.$ Lys $^{221}$, Lys $^{224}$, Lys $\left.^{225}\right)$ and the region of lysine 270

$237\left(\right.$ Lys $^{274}$, Lys $^{275}$, and Lys $\left.{ }^{279}\right)$ had different deuteration profiles. The 220 lysine

238 residues had a smaller decrease in deuterium incorporation than the 270 lysine

239 residues (Figure $\mathbf{8 C}$ ). The residues between 221 and 229 have previously been

240 shown to interact with PS, and the HDX supports these observations as the

241 deuterium exchange was reduced for PS containing vesicles slightly greater than 


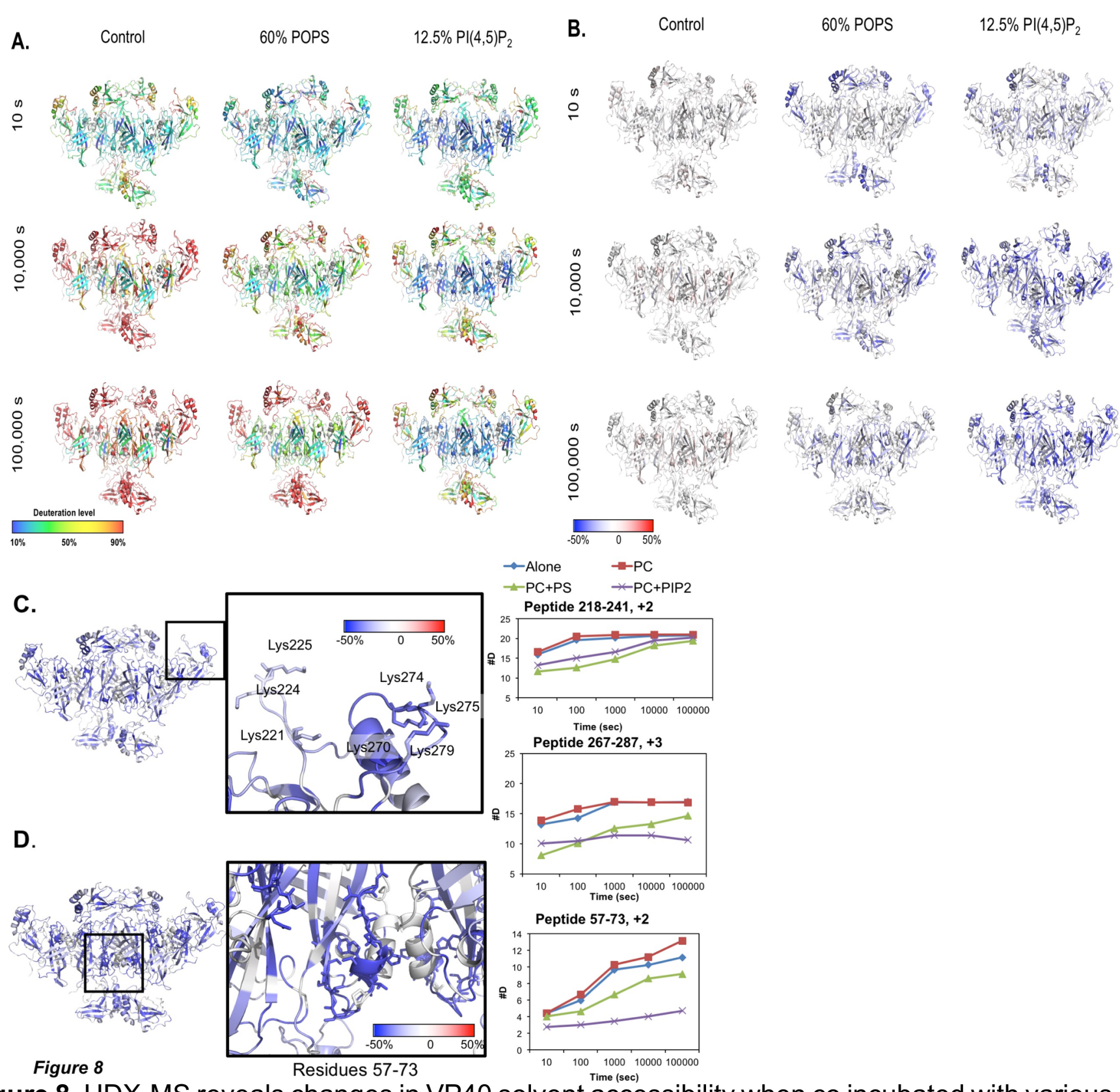

Figure 8. HDX-MS reveals changes in VP40 solvent accessibility when co incubated with various liposomes. A. Deuteration level of VP40 (shown as the hexamer) at $10 \mathrm{~s}, 10,000 \mathrm{~s}$ and 100,000 $\mathrm{s}$ time points after a 30 minute incubation with Control liposomes, PS containing liposomes, or $\mathrm{PI}(4,5) \mathrm{P}_{2}$ containing liposomes. B. The influence of Control liposomes, $\mathrm{PS}$ containing liposomes, or $\mathrm{PI}(4,5) \mathrm{P}_{2}$ containing liposomes on VP40 deuteration is mapped onto the VP40 hexamer structure for the $10 \mathrm{~s}, 10,000 \mathrm{~s}$ and $100,000 \mathrm{~s}$ time points. C. The influence of $\mathrm{PI}(4,5) \mathrm{P}_{2}$ containing liposomes on VP40 $\mathrm{PI}(4,5) \mathrm{P}_{2}$ binding residues is enlarged. Deuterium incorporation in these regions is plotted in the peptide map to the right of the region of interest. The y-axis is the number of deuterium incorporated into the peptide fragment with respect to time on the $x$-axis. $\mathbf{D}$. The influence of $\mathrm{PI}(4,5) \mathrm{P}_{2}$ containing liposomes on VP40 $\mathrm{PI}(4,5) \mathrm{P}_{2}$ residues hypothesized to be important for the stabilization of large oligomers (residues 57-73). Deuterium incorporation in these regions is plotted in the peptide map to the right of the region of interest.

242 that for $\mathrm{PI}(4,5) \mathrm{P}_{2}$. In contrast, the region containing key Lys residues in $\mathrm{PI}(4,5) \mathrm{P}_{2}$ 
243 binding $\left(\right.$ Lys $^{270}$, Lys $^{274}$, Lys $^{275}$, and Lys $\left.{ }^{279}\right)$ had a slower rate of exchange with

244 deuterium with either $\mathrm{PS}$ or $\mathrm{PI}(4,5) \mathrm{P}_{2}$ vesicles, with $\mathrm{PI}(4,5) \mathrm{P}_{2}$ vesicles more

245 significantly reducing deuterium exchange over the time course of the interaction.

$246 \mathrm{PI}(4,5) \mathrm{P}_{2}$ had a significant affect in slowing the exchange in this CTD region,

247 which correlates with the important role of Lys ${ }^{274}$, Lys $^{275}$, and Lys ${ }^{279}$ in VLP

248 formation and formation of large VP40 oligomers (Figure 6-7).

249 The most dramatic changes observed were seen in the NTD residues 57-

250 73, a novel site likely important for VP40 oligomer stability. Residues 57-73 are

251 seen packed against the $\mathrm{N}-\mathrm{N}$ terminal alpha helical interface (Figure 8D). The

$252 \mathrm{~N}-\mathrm{N}$ terminal dimer interface is retained in the hexamer structure; this structure is

253 extremely stable and no deuterium is incorporated even after $100,000 \mathrm{~s}$ under all

254 conditions (Figure S5-S10). $\mathrm{PI}(4,5) \mathrm{P}_{2}$ and $\mathrm{PS}$ both significantly reduced

255 deuterium incorportation in this $\mathrm{N}$-terminal domain oligomerization interface

256 (residues 57-73), with $\mathrm{PI}(4,5) \mathrm{P}_{2}$ providing greater stability over the lifetime of the

257 experiments again underscoring the importance of $\mathrm{PI}(4,5) \mathrm{P}_{2}$ in VP40 hexamers

258 and large oligomers.

259 Molecular Dynamics Section

260 To gain more molecular insight into $\mathrm{PI}(4,5) \mathrm{P}_{2}$ coordination by VP40, we

261 performed molecular dynamics simulations of VP40 dimer and hexamers with

262 bilayers containing $\mathrm{Pl}(4,5) \mathrm{P}_{2} . \mathrm{PI}(4,5) \mathrm{P}_{2}$ was shown to cluster at the ends of the

263 VP40 hexamers where interactions between Lys residues in CTD regions 1 and

2642 occurred (Figure 9). The H-bonding was monitored over the lifetime of the

265 simulations using an asymmetric bilayer. The lipid composition for the $\mathrm{PIP}_{2}$ 

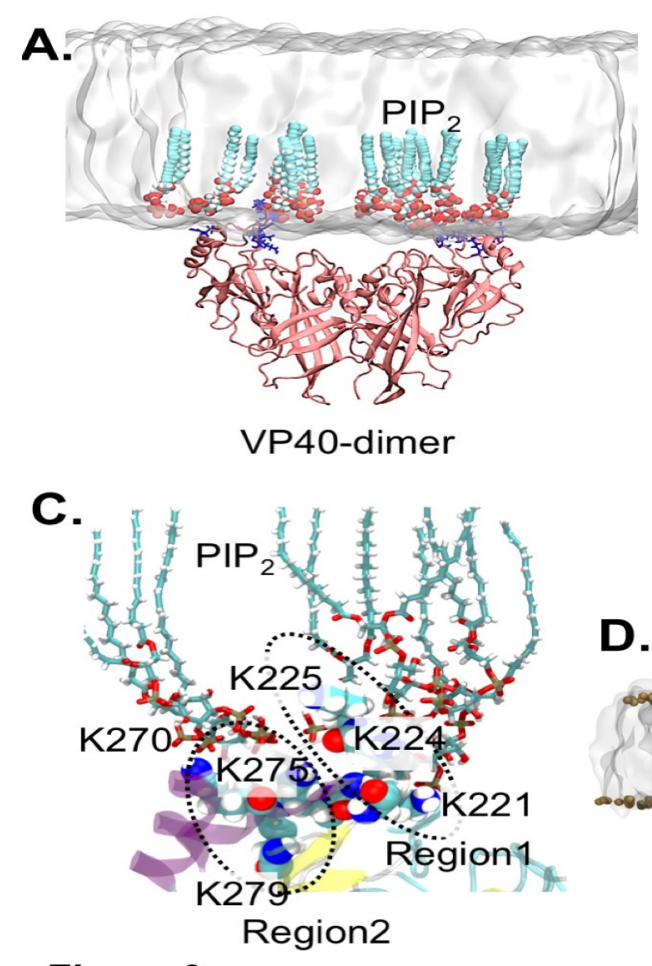

Figure 9
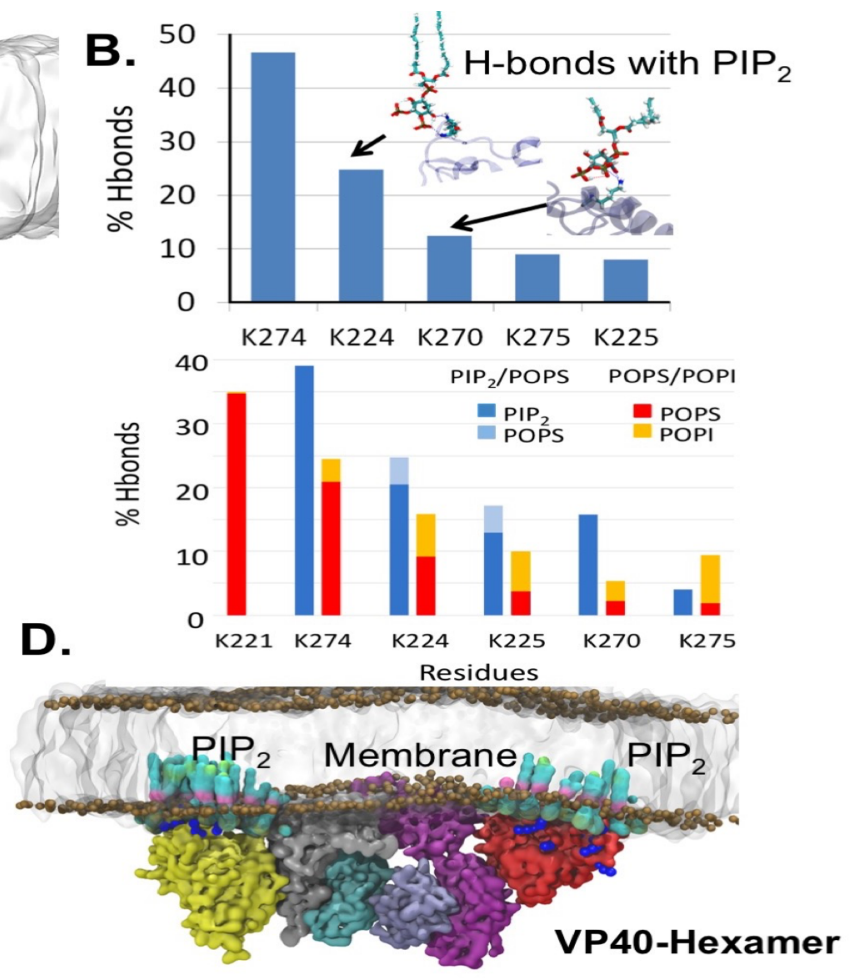

Figure 9. Molecular Dynamics of VP40-PIP ${ }_{2}$ interactions. A. VP40 dimer interacting with the PM containing PIP2 lipids. B. Hydrogen bonding between the CTD-residues and the $\mathrm{PIP}_{2}$ head-groups (top panel) and the CTD-residues and PS, $\mathrm{PIP}_{2}$ or other lipids (bottom panel) for the VP40 dimer. C. Lysine residues in region 1 and region 2 interacting with $\mathrm{PIP}_{2}$. D. Coarse-grained model showing VP40 hexamer interacting with the PM. The $\mathrm{PIP}_{2}$ lipids interacting with the VP40 are highlighted. containing membrane was in the ratio (21:11:32:17:9:10)

267 (CHOL:POPC:POPE:POPS:PIP $2: P S M)$ for the inner leaflet of the PM and the

268 outer leaflet had the ratio (19:33:8:3:4:33). Lys ${ }^{274}$ made the most $\mathrm{H}$-bonds over

269 the simulation time with $\mathrm{PI}(4,5) \mathrm{P}_{2}$ whereas key $\mathrm{H}$-bonds were also found for

270 Lys $^{224}$, Lys ${ }^{225}$, Lys $s^{270}$ and Lys ${ }^{275}$. In contrast, Lys ${ }^{221}$ made H-bond contacts with

271 PS only as opposed to $\mathrm{PI}(4,5) \mathrm{P}_{2}$, which is supported by recent identification of

272 the VP40 PS binding site ${ }^{22}$ suggesting reduction in $\mathrm{PI}(4,5) \mathrm{P}_{2}$ binding by this

273 mutant may be due to reduced electrostatic interactions. Coarse-grained MD

274 simulations were performed with the VP40 hexamer and an asymmetric bilayer 
275 consisting of (POPC:POPE:PSM:POPS:PIP $2: C H O L)$ in the ratio (41:8:23:4:4:20)

276 on the upper leaflet and (11:37:5:16:10:21) on the lower leaflet (Fig. 9D). Again,

277 Lys221 made few contacts with $\mathrm{PI}(4,5) \mathrm{P} 2$ throughout the 1000 ns simulation time

278 whereas significant contacts were observed between $\mathrm{PI}(4,5) \mathrm{P} 2$ and Lys ${ }^{274}$,

279 Lys $^{270}$, Lys $^{224}$, Lys $^{225}$, and Lys ${ }^{275}$ (Fig. S14).

280 VP40 budding is reduced when $\mathrm{PI}(4,5) \mathrm{P}_{2}$ is depleted with small molecule

281 inhibitors:

282 To determine if pharmacological inhibition of $\mathrm{PI}(4,5) \mathrm{P}_{2}$ synthesis was a

283 feasible approach to inhibit VLP formation, we employed cellular treatments with

284 Phenylarsine oxide (PAO) and Quercetin (Q), which have been used to suppress

$285 \mathrm{PI}(4,5) \mathrm{P}_{2}$ synthesis ${ }^{34}$. Cell viability was assessed with various concentrations of

286 each drug using the Cell TiterGlo assay kit (see Methods section). Q is a natural

287 product found in many foods ${ }^{35-39}$ and has been reported as a tyrosine

288 phosphatase inhibitor ${ }^{40}$, lipid kinase inhibitor ${ }^{34}$, as well as an anti-microbial,

289 pancreatic lipase inhibitor, and HIV inhibitor ${ }^{41}$. We are interested in the PI5

290 kinase inhibitory effects of $\mathrm{Q}$, which was shown to inhibit $\mathrm{PI}(4,5) \mathrm{P}_{2}$ synthesis at

291 the $\mathrm{PM}^{34}$. Additionally, HIV-GAG requires $\mathrm{PI}(4,5) \mathrm{P}_{2}$ for efficient virus particle

292 release and a $40 \mu \mathrm{M}$ treatment of cells with $\mathrm{Q}$, resulted in $80 \%$ inhibition of viral

293 spread $^{41}$. PAO was also shown to inhibit $\mathrm{PI}(4,5) \mathrm{P}_{2}$ synthesis though inhibition of

294 Pl4 kinase IIIa ${ }^{34}$. Previously, we found that a $10 \mu \mathrm{M}, 30$ minute PAO treatment

295 significantly reduced VP40 localization from the $\mathrm{PM}^{26}$. 
297 over a longer treatment time. Confocal imaging of PLC $\mathrm{P}-\mathrm{PH}$ with each treatment

298 showed a significant but not complete depletion of $\mathrm{PI}(4,5) \mathrm{P}_{2}$ from the $\mathrm{PM}$ of cells

299 with $500 \mathrm{nM}$ PAO or $100 \mu \mathrm{M}$ Q compared to the respective DMSO vehicle

300 controls (Figure 10A). The population of cells transfected with VP40 that formed

A.
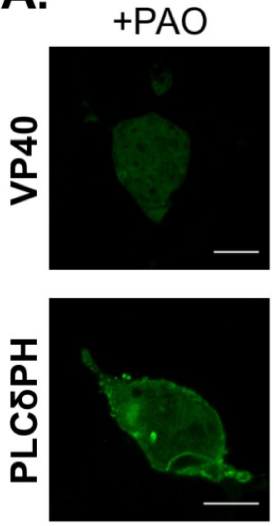

C.
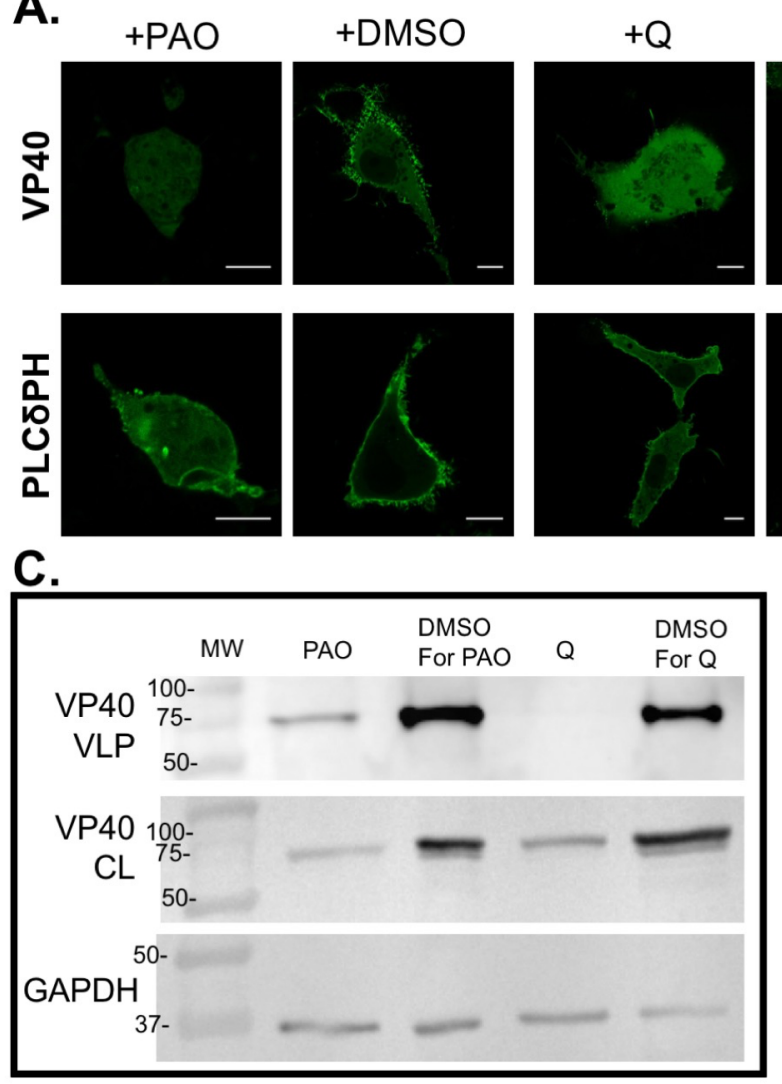

D.

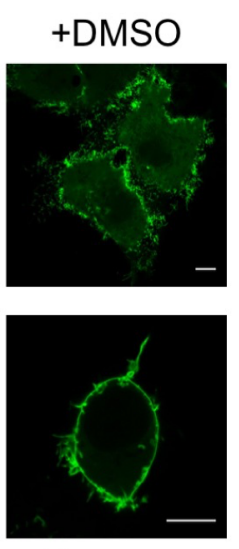

B.

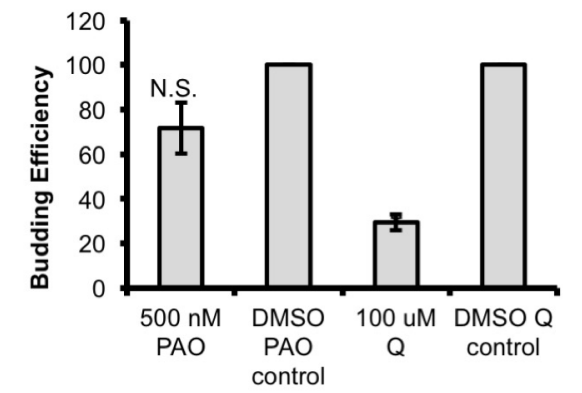

Figure 10

Figure 10: Kinase inhibitors reduce VP40 budding. A. Representative images of PLCסPH-EGFP and WT-VP40-EGFP 12 hour post treatment with $500 \mathrm{nM}$ PAO or $100 \mu \mathrm{M}$ Quercetin (Q). Scale bars are $10 \mu \mathrm{m}$. B. Cell populations with VLP expression (for VP40) and complete PM localization (for PLCঠPH). Values are normalized to cells treated with the equivalent volume of DMSO as a loading control. C. Western blots Western blots of WT-VP40-EGFP show VP40 in the VLP and the cell lysate $(C L)$ fractions for each treatment condition, equivalent volumes of DMSO are used as a control for each treatment. GAPDH, was used as a loading control for these experiments. Molecular weight markers are shown for all blots to verify band size for VP40-EGFP and GAPDH. D. Budding efficiency is normalized to the DMSO controls. Average values are shown \pm the SEM, ${ }^{*} \mathrm{P}<0.05$.

301 VLPs was also reduced significantly (Figure 10A). Next, VLPs and CL samples 
302 were collected and the percent budding was determined with western blot

303 analysis for PAO and Q treatments (Figure 10C and 10D). The VP40 expression

304 was lower in PAO and Q treated cells but the loading control, GAPDH, did not

305 show changes in expression. PAO treated cells had a $\sim 30 \%$ decrease in budding

306 compared to the DMSO control, which was not statistically significant over three

307 independent VLP collections. Higher concentrations of PAO are used for more

308 complete $\mathrm{PI}(4,5) \mathrm{P}_{2}$ depletion in cells, however, these concentrations were toxic

309 to cells over longer treatment times (Figure S12). Q treated cells had a

310 statistically significant decrease in budding when compared to the DMSO control.

$311 \mathrm{Q}$ treatment in Phase I clinical trials recommended a $1400 \mathrm{mg} / \mathrm{m}^{2}$ intravenous

312 treatment ${ }^{42}$. Because PLCס-PH showed that not all PM PI(4,5) $\mathrm{P}_{2}$ is depleted at

313 this concentration of $Q$, we hypothesize additional $Q$ effects are resulting in the

314 more dramatic decrease in VP40 VLP budding than with PAO treatment.

315 Additional research will prove useful to more closely investigate the mechanism

316 of Q on VP40 function especially as this molecule is a natural product with low

317 toxicity.

319 Discussion:

320 VP40 binds selectively to $\mathrm{PI}(4,5) \mathrm{P}_{2}$ over the other seven

321 phosphatidylinositol lipids (Figure 2A and 2B). The apparent affinity for

$322 \mathrm{PI}(4,5) \mathrm{P}_{2}$ containing LUVs is approximately $170 \mathrm{nM}$ (Figure 2C) and this affinity

323 would likely be increased though avidity with VP40 oligomers and likely $\mathrm{PI}(4,5) \mathrm{P}_{2}$

324 induced clustering in the cellular environment ${ }^{28}$. Binding of $\mathrm{VP} 40$ to $\mathrm{PI}(4,5) \mathrm{P}_{2}$ is 
325 mediated in part by lysine residues in region 1 (K221, K224, K225) and region 2

326 (K270, K274, K275, K279), which contributed to plasma membrane localization,

327 VP40 oligomerization, and budding (Figures 3-7). Interestingly, these two

328 binding regions have distinct VP40 phenotypes. For example, region 1 lysine

329 residues are required for the formation for oligomers larger than a hexamer

330 whereas region 2 lysine residues harbor more significantly reduced VLP

331 formation but didn't have as a profound of an effect on the formation of large

332 VP40 oligomers. This data is in agreement with the confocal imaging and

333 assessment of cell populations with VLPs (Figure 4) and SEM imaging (Figure

334 5).

335 The HDX MS data confirmed these VP40 binding sites are distinct as they

336 have different deuterium incorporations when bound to $\mathrm{PI}(4,5) \mathrm{P}_{2}$ liposomes. HDX

337 MS was recently used to study Marburg virus VP40 (mVP40) oligomerization and

338 lipid binding ${ }^{43}$. mVP40 interacts with anionic membranes through electrostatic

339 interactions ${ }^{44}$ and HDX MS confirmed that these interactions occur via CTD

340 residues and that mVP40 undergoes oligomer formation when bound to anionic

341 membranes. In contrast to eVP40, the lifetime of the mVP40 interactions with

342 anionic membranes was relatively short ${ }^{43}$. In region 1 of VP40, PS contributed to

343 a slower exchange rate with deuterium than that of $\mathrm{PI}(4,5) \mathrm{P}_{2}$ and this region was

344 more important for formation of VP40 oligomers larger than a hexamer.

345 Previously, region 1 was shown to interact with $\mathrm{PS}^{22}$ and PS was necessary for

346 formation for formation of VP40 oligomers larger than hexamers ${ }^{21}$. Thus, region

3471 may be able to bind $\mathrm{PS}$ or $\mathrm{PI}(4,5) \mathrm{P}_{2}$ with $\mathrm{PS}$ providing an interaction that is 
348 able to induce VP40 dimer conformational change to a greater extent. More

349 significant exploration of dynamic and structural consequences of lipid head

350 group binding by VP40 will be necessary to delineate the molecular basis of

351 these conformational transitions.

352 In region $2, \mathrm{PI}(4,5) \mathrm{P}_{2}$ slowed exchange more significantly than $\mathrm{PS}$,

353 indicating a more stable VP40 complex. Residues in region 2 had differential

354 effects on VP40 dependent properties depending upon the assay. Lys ${ }^{274}$ seems

355 to be a key $\mathrm{PI}(4,5) \mathrm{P}_{2}$ interacting residue, which was supported by the MD

356 simulations. Lys 275 also made important contributions to $\mathrm{Pl}(4,5) \mathrm{P} 2$ binding in in

357 vitro and in silico assays and like K274A, K275A reduced VP40 oligomerization

358 and VLP formation. The slight differences between K274A and K275A in the

359 assays could be partially attributed to a role of K275A in intermolecular VP40

360 interactions which decrease the ability of Lys ${ }^{275}$ to be free for membrane binding

361 prior to association with the membrane surface (Fig. S15). K270 and K279 in

362 region 2 also play a significant role in aspects of $\mathrm{PI}(4,5) \mathrm{P}_{2}$ mediated budding as

363 both K270A and K279A mutants show reduced binding to LUVs and K279A

364 nearly completely abrogated VLP formation. In contrast, K279A large oligomers

365 formed similar to WT suggesting the contacts K279A helps facilitate with the

366 membrane or among VP40 oligomers is critical to budding. Despite a reduction

367 in $\mathrm{Pl}(4,5) \mathrm{P}_{2}$ binding and VP40 oligomerization, K270A was sufficient for

368 approximately the same budding efficiency as WT.

$369 \mathrm{~K} 104 \mathrm{~A}$ is an interesting mutation as it has VP40 octamer like tendencies in

370 binding (see Figures 3 and S13) and forms an abundance of the octamer 
371 conformation when purified from bacteria compared to WT VP40 as seen by size

372 exclusion chromatography (data not shown). K104A sits at the $\mathrm{N}-\mathrm{C}$ terminal

373 domain interface and may be an important residue involved in the unlatching

374 process during self-oligomerization. K104A did have a slight yet non-significant

375 increase in larger oligomers as determined with N\&B analysis indicating it may

376 promote larger oligomer formation. Together, our data suggests that K104 is

377 important for stabilization of the dimer $\mathrm{N}-\mathrm{C}$ terminal domain latch, as there is an

378 increase in binding to neutral lipids with the K104A mutant. Removal of this lysine

379 may result in a destabilized dimer conformation and promote premature

380 oligomerization that results in a significant decrease in budding. Increasing VP40

381 PM localization and oligomerization does not always indicate that it will promote

382 budding ${ }^{45}$. When VP40 inter-domain dynamics were investigated by GC et al.,

383 mutants that promoted PM localization did not show a significant increase in VLP

384 budding $^{45}$. Further investigation of the role of $\mathrm{K} 104$ and the potential salt bridge

385 between K104 and D193 may reveal a novel VP40 oligomerization mechanism.

386 We hypothesize that binding to negatively charged $\mathrm{PI}(4,5) \mathrm{P}_{2}$ or $\mathrm{PS}$ at this site

387 could initiate the unlatching of the potential K104-D193 salt bridge.

388 PAO and Q treatments reduced the overall VP40 expression levels but $Q$

389 had a significant effect on VLP formation whereas PAO did not. This observation

390 raises the question whether VP40 requires $\mathrm{PI}(4,5) \mathrm{P}_{2}$ for efficient expression and

391 stability. VP40 enters the nucleus during viral infection ${ }^{19}$ and there is a pool of

392 nuclear $\mathrm{PI}(4,5) \mathrm{P}_{2}$ that is not completely understood ${ }^{46}$. Alternatively, there is a

393 self-inhibitory effect on VP40 expression if VP40 membrane binding cannot be 
394 achieved. The VP40 octamer formation and RNA binding event is critical for viral

395 success, but the VP40 RNA sequence specificity is still unknown. Additionally,

396 the role of VP40 octamer in the nucleus is undetermined but found to be

397 essential for viral success ${ }^{18}$. Additional studies will enhance this mysterious

398 aspect of VP40 function and could provide novel EBOV inhibitory pathways and

399 mechanisms. There are many open questions remaining about the function of the

400 VP40 octamer in the viral lifecycle.

401 Quercetin treatment shows promise for an Ebola treatment. Could this

402 molecule be adapted for higher potency without an increase in cellular toxicity?

403 Here we found that $100 \mu \mathrm{M} Q$ treatment and $500 \mathrm{nM}$ PAO treatment result in a

404 decrease in $\mathrm{PI}(4,5) \mathrm{P}_{2}$ at the plasma membrane in consonance with previous

405 reports ${ }^{34}$. Interestingly, $Q$ had a greater inhibitory effect on budding than PAO.

406 We hypothesize the difference in VLP budding with $Q$ treatment may be from

407 additional inhibitory effects beyond $\mathrm{PI}(4,5) \mathrm{P}_{2}$ reduction such as tyrosine

408 phosphatase inhibition. Gatto et al. found an $80 \%$ reduction in HIV-1 with a 40

$409 \mu \mathrm{M}$ treatment and found that the inhibitory effects were related to the presence of

410 the C-3 hydroxyl group on $\mathrm{Q}^{41}$. Additional research testing other flavonoid

411 molecules against VP40 (BSL 2) or the Ebola virus (BSL 4) could provide

412 powerful insights to viral inhibition. Because $Q$ is a natural product found in

413 several common foods and deemed safe by the FDA as a food additive up to 500

414 mg per serving, we believe it shows significant promise for testing as a

415 therapeutic against virus replication. 
417 Methods:

418 Molecular Biology. Primers were designed for single and double VP40 mutants

419 and synthesized by Integrated DNA Technologies (Coralville, lowa). The

420 following primers were used: K104A: Forward CCT CTA GGT GTC GCT GAT

421 CAA GCG ACC TAC AGC TTT GAC TC, Reverse GAG TCA AAG CTG TAG

422 GTC GCT TGA TCA GCG ACA CCT AGA GG. K221A: Forward GC CCC ATT

423 CTT TTA CCC AAC GCA AGT GGG AAG AAG GGG AAC, Reverse GTT CCC

424 CTT CTT CCC ACT TGC GTT GGG TAA AAG AAT GGG GC. K224A: Forward

425 CCC AAC AAA AGT GGG GCG AAG GGG AAC AGT GCC GAT CTA ACA TCT

426 CCG G, Reverse CCG GAG ATG TTA GAT CGG CAC TGT TCC CCT TCG

427 CCC CAC TTT TGT TGG G. K225A: Forward CCC AAC AAA AGT GGG AAG

428 GCG GGG AAC AGT GCC GAT CTA ACA TCT CCG G, Reverse CCG GAG

429 ATG TTA GAT CGG CAC TGT TCC CCG CCT TCC CAC TTT TGT TGG G.

430 K236A: Forward GCC GAT CTA ACA TCT CCG GAG GCA ATC CAA GCA ATA

431 ATG ACT TCA C, Reverse GTG AAG TCA TTA TTG CTT GGA TTG CCT CCG

432 GAG ATG TTA GAT CGG C. H269A: Forward G CCA GAA ACT CTG GTC GCC

433 AAG CTG ACC GGT AAG AAG G, Reverse CCT TCT TAC CGG TCA GCT TGG

434 CGA CCA GAG TTT CTG GC. K270A: Forward G CCA GAA ACT CTG GTC

435 CAC GCG CTG ACC GGT AAG AAG G, Reverse CCT TCT TAC CGG TCA

436 GCG CGT GGA CCA GAG TTT CTG GC. K274A: Forward G GTC CAC AAG

437 CTG ACC GGT GCG AAG GTG ACT TCT AAA AAT GG, Reverse CCA TTT

438 TTA GAA GTC ACC TTC GCA CCG GTC AGC TTG TGG ACC. K275A:

439 Forward G GTC CAC AAG CTG ACC GGT AAG GCG GTG ACT TCT AAA AAT 
440 GG, Reverse CCA TTT TTA GAA GTC ACC GCC TTA CCG GTC AGC TTG

441 TGG ACC. K279A: Forward CC GGT AAG AAG GTG ACT TCT GCA AAT GGA

442 CAA CCA ATC ATC CC, Reverse GGG ATG ATT GGT TGT CCA TTT GCA

443 GAA GTC ACC TTC TTA CCG G. K291A: Forward C CCT GTT CTT TTG CCA

444 GCG TAC ATT GGG TTG GAC CCG, Reverse CGG GTC CAA CCC AAT GTA

445 CGC TGG CAA AAG AAC AGG G. K224A/K225A: Forward CCC AAC AAA

446 AGT GGG GCG GCG GGG AAC AGT GCC GAT CTA ACA TCT CCG G,

447 Reverse CCG GAG ATG TTA GAT CGG CAC TGT TCC CCG CCG CCC CAC

448 TTT TGT TGG G. K274/K275A (made from K274A template): Forward GGT

449 CCA CAA GCT GAC CGG TGC GGC GGT GAC TTC TAA AAA TGG, Reverse

450 CCA TTT TTA GAA GTC ACC GCC GCA CCG GTC AGC TTG TGG ACC. Site

451 directed mutagenesis was performed using a Quikchange XL cloning kit as

452 described by the manufacturer (Agilent Technologies, Santa Clara, CA). Sanger

453 sequencing was used to verify mutagenesis (Notre Dame Genomics Facility).

454 Protein Purification. VP40-6xHis-pET46 Ek/LIC was expressed in Rosetta BL21

455 DE3 cells (EMD Millipore Corp, Billerica, MA) as previously described ${ }^{18}$. Briefly,

456 cells were grown to $\mathrm{OD}_{600}$ of 0.6 then induced with $1 \mathrm{mM}$ IPTG for 5 hours at RT.

457 Protein was purified with a Ni-NTA affinity column followed by size exclusion

458 chromatography (HiLoad Superdex 200 pg). Protein concentration was

459 determined by BCA assay (Thermo Fisher Scientific, Waltham, MA) and protein

460 was stored at $4^{\circ} \mathrm{C}$ for up to two weeks.

461 Liposome Pelleting Assay. All lipids were purchased from Avanti Polar Lipids

462 (Alabaster, AL). The liposome pelleting assay was adapted from Julkowska et 
463 al. $^{30}$. Lipid films were hydrated with fresh raffinose buffer (250 mM Raffinose

464 pentahydrate, $150 \mathrm{mM} \mathrm{NaCl}, 10 \mathrm{mM}$ Tris, $\mathrm{pH} 8.0$ ) by thorough heating to $37^{\circ} \mathrm{C}$

465 and vortexing. Resuspended lipids were then extruded through a $200 \mathrm{~nm}$ filter

466 with an Avanti lipid extruder. Dynamic light scattering (Delsa Nano S particle size

467 analyzer, Brea, CA) was used to confirm liposome size. Liposomes were then

468 diluted and added to the reaction at a final concentration of $1.6 \mathrm{mM}$ lipid and 3.3

469 uM protein. Reaction mixtures were incubated at room temperature for 30

470 minutes then centrifuged at $75,000 \times g$ for 30 minutes at $22^{\circ} \mathrm{C}$.

471 Surface plasmon resonance (SPR). SPR measurements were made using a

472 Biacore X (GE Healthcare) with an L1 sensor Chip (GE Healthcare). Liposomes

473 were prepared by hydrating lipid films in $10 \mathrm{mM}$ TRIS, pH 7.4 containing $150 \mathrm{mM}$

$474 \mathrm{NaCl}$ followed by extrusion through a $100 \mathrm{~nm}$ filter. All experiments were

475 performed at room temperature in $150 \mathrm{mM} \mathrm{NaCl}, 10 \mathrm{mM}$ TRIS, pH 7.4 using the

476 method previously described in detail ${ }^{32}$. The lipid composition used was

477 POPC:DOPE (80:20) on flow cell 1 and POPC:DOPE:PI(4,5)P 2 75:20:5 on flow

478 cell 2. The DPPC:Cholesterol coating had a low response so was not used

479 (data not shown). The apparent affinity $\left(K_{d}\right)$ was determined in Kaleidagraph

480 with the following equation: $R_{e q}=R_{\max } /\left(1+K_{d} /[P]\right)$ where $[P]$ is protein

481 concentration and $\mathrm{R}$ is response units.

482 Cell Culture and transfection. COS-7 cells were maintained in a humidified

483 chamber at $37^{\circ} \mathrm{C}$ and $5 \% \mathrm{CO}_{2}$. The cells were kept in DMEM with L-Glutamine,

484 D-Glucose and Sodium Pyruvate (Life Technologies, Carlsbad, CA) with 10\%

485 FBS (Sigma, St. Louis, MO) and 1\% Penicillin-Streptomycin (Life Technologies) 
486 at $37^{\circ} \mathrm{C}, 5 \% \mathrm{CO}_{2}$. For imaging, cells were seeded in 8-well imaging plates

487 ((MatTek, Ashland, MA) and transfected with Lipofectamine 2000 or

488 Lipofectamine LTX with Plus reagent (Life Technologies), 0.4 ug DNA in

489 optiMEM (Life Technologies) for 12-14 hours. Transfection reactions were scaled

490 up proportionally for scanning electron microscopy and VLP experiments.

491 Confocal Imaging. A Zeiss 710 laser scanning microscope was used to image

492 EGFP-VP40 phenotype in COS-7 cells. A $488 \mathrm{~nm}$ laser was used to excite

493 EGFP. All EGFP-VP40 expressing cells were counted as either presenting VLPs

494 or not were counted. VP40-EGFP mutants were compared to WT. Each

495 construct was counted and imaged over at least three independent experiments.

496 For number and brightness experiments, an Olympus FV1000 instrument was

497 used according to the procedure explained in depth previously ${ }^{47}$. Data analysis

498 was performed in SimFCS software (Globals Software, Irvine, CA) as previously

499 described in detail ${ }^{47}$.

500 Scanning Electron Microscopy. Transfected cells were scraped from plates 12-

50114 hours post transection as described previously in detail ${ }^{31}$. Images were

502 acquired on a Field Emission Scanning Electron Microscope Magellan 400 (FEI,

503 Hillsboro, OR) at the Notre Dame Integrated Imaging Facility (NDIIF).

504 VLP Collection and Western Blot Analysis. VLPs were harvested 24 hours

505 post transfection as described previously in detail ${ }^{31}$. Briefly, VLPs were washed

506 from cells with 1X PBS, and applied to a sucrose cushion. Samples were

507 centrifuged at $100,000 \times g$, at $4^{\circ} \mathrm{C}$ for 2 hours. During the centrifugation, cells

508 were scraped from plates and lysed with RIPA buffer. Soluble protein was 
509 isolated from cell lysate via centrifugation at $17,000 \times g$ at $4^{\circ} \mathrm{C}$ for 15 minutes,

510 followed by removal of the soluble fraction of samples. Samples were then

511 stored at $-80^{\circ} \mathrm{C}$. A BCA assay was used to determine protein concentration of all

512 samples, 15 ug of each cell lysate was loaded for SDS PAGE and Western Blot.

513 A proportional volume of the VLP sample was loaded for SDS PAGE and

514 Western Blot. For VP40 detection, mouse anti-EGFP (F56-6A1.2.3,

515 ThermoFisher Scientific) primary antibody and sheep anti-mouse HRP (AB 6808,

516 Abcam, Cambridge, United Kingdom) secondary antibody was used. For GAPDH

517 detection, an anti-GAPDH antibody was used (AB 8245, Abcam) and sheep anti

518 mouse HRP (AB 6808, Abcam) secondary antibody was used. ECL blotting

519 substrate was used according to the manufactures instructions (Thermo

520 Scientific). Blots were imaged on a GeneGnome (Syngene, India). ImageJ was

521 used to quantify band density and determine budding efficiency.

522 Hydrogen Deuterium Exchange Mass Spectrometry (HDX MS). HDX MS

523 experiments were performed as described in detail previously with Marburg VP40

$524 \quad 43$. For Ebola VP40, multilamellar vesicles (MLVs) of the following compositions

525 were used: PC (50\% POPC, 50\% DOPE), 12.5\% PI(4,5)P 2 (47.5\% POPC, 40\%

526 DOPE, $\left.12.5 \% \mathrm{PI}(4,5) \mathrm{P}_{2}\right)$, and 60\% POPS (30\% POPC, 10\% DOPE, 60\%

527 POPS). 3 ug protein and $2.8 \mathrm{mM} \mathrm{MLV}$ were incubated 30 minutes prior to the

528 HDX experiments. HDX data was analyzed as previously described in detail ${ }^{43}$

529 and mapped to eVP40 dimer (4ldb) and hexamer (4Ldd with modeled C-terminal

530 domains courtesy of Dr. Prem Chapagain ${ }^{28}$ ). 
531 Cell Viability Assay. Cell viability was determined using the CellTiter Glo assay

532 (Promega, Madison, WI) according to the manufactures instructions. Cells were

533 treated with indicated therapeutics for 24 hours in optiMEM (Life Technologies).

534 The viability assay was performed at least two independent times for each drug

535 concentration or equivalent volume of vehicle, DMSO. Drug concentrations that

536 allowed for $80 \%$ cell viability or more were tested further in imaging and VLP

537 collection experiments.

538 Pharmacological Treatments. Phenyl arsine oxide (PAO) (Sigma) and

539 Quercetin $(Q)$ (Sigma) stocks were prepared in DMSO and diluted in OptiMEM

540 (Life Technologies) prior to use. For imaging experiments, cells were treated with

541 PAO or $\mathrm{Q}$ for 14 hours after being added 4 hours post transfection. For VLP

542 collection experiments, cells were transfected with Lipofectamine LTX (Life

543 Technologies) then treated with PAO or $\mathrm{Q}$ for 20 . In both experiments,

544 transfection media was removed with aspiration and replaced with drug or DMSO

545 containing optiMEM. The equivalent volume of DMSO was added as a vehicle

546 control for PAO and Quercetin imaging and VLP collection experiments. Each

547 imaging experiment and VLP collection was performed three independent times.

549 Reproducibility and Statistics. At least three experimental replicates were

550 performed for all experiments unless otherwise noted. A two tailed students T-

551 test was used to determine statistical differences. A P value of 0.05 or less was

552 considered statistically significant. Error bars are shown as the standard error of

553 the mean in all graphs unless otherwise noted. 
555 All-atom simulations of VP40 Dimer and Plasma Membrane. The crystal

556 structure of VP40 dimer was obtained from the Protein Data Bank (PDB ID:

557 4LDB). The protein and plasma membrane (PM) complex was set up ${ }^{48}$ using the

558 Charmm-Gui web server ${ }^{49}$. The PM systems consisted of different types of lipids:

559 1-palmitoyl-2-oleoyl-sn-phosphatidylcholine (POPC), 1-palmitoyl-2-oleoyl-sn-

560 phosphatidyl-ethanolamine (POPE), 1-palmitoyl-2-oleoyl-sn-phosphatidyl- serine

561 (POPS), palmitoylsphingomyelin (PSM), 1-palmitoyl-2-oleoyl-sn-glycero-3-

562 phosphoinositol (POPI), palmitoyl-oleoyl-phosphatidylinositol-(4,5)-bisphosphate

$563\left(\mathrm{PIP}_{2}\right)$ and cholesterol (CHOL). Two different systems were constructed: one with

564 PIP2 and another with POPI. The lipid composition for the $\mathrm{PIP}_{2}$ containing

565 membrane was in the ratio $(21: 11: 32: 17: 9: 10)$

566 (CHOL:POPC:POPE:POPS:PIP2:PSM) for the inner leaflet of the PM and the

567 outer leaflet had the ratio (19:33:8:3:4:33). There were 442 lipids in the outer

568 leaflet and 451 on the inner leaflet. A similar membrane was constructed

569 containing POPI with a lipid composition ratio of (21:11:32:17:9:10) (CHOL:

570 POPC: POPE: POPS: POPI: PSM) for the inner leaflet and (19:32:8:3:4:34) for

571 the outer leaflet. There were 149 lipids in outer leaflet and 151 in the inner leaflet

572 for this system.

$573 \quad$ Both dimer-membrane systems were solvated using TIP3 water

574 molecules. The total system consists of around 361000 atoms (PIP 2 setup) and

575 around 121,000 atoms for (POPI set up). We performed all-atom molecular

576 dynamics simulations using NAMD2.10 ${ }^{50}$ with the CHARMM36 force field for both

577 systems. The particle mesh Ewald (PME) method was used in order to treat long 
578 range electrostatic interaction and the SHAKE algorithm was employed to

579 constrain hydrogen-containing covalent bonds. The system was minimized for

58010,000 steps followed by a six step equilibration process. Pressure was

581 controlled using a Nose-Hoover Langevin-piston method. Langevin temperature

582 coupling with a friction coefficient of $1 \mathrm{ps}^{-1}$ was used to control the temperature

583 and a 2 fs time step was used for production runs.

584

585 Coarse-grained VP40 Hexamer and Plasma Membrane. The crystal structure

586 of the VP40 hexamer was obtained from the Protein Data Bank (PDB: 4LDD).

587 Missing residues were added using the Modeller software package. The plasma

588 membrane and hexamer system was built ${ }^{28}$ using the Charmm-gui webserver ${ }^{49}$.

589 The composition of the various lipids (POPC:POPE:PSM:POPS:PIP $:$ :CHOL)

590 were in the ratio $(41: 8: 23: 4: 4: 20)$ on the upper leaflet and $(11: 37: 5: 16: 10: 21)$ on

591 the lower leaflet. Since the total number of atoms was greater than 600,000

592 atoms, a coarse grained (CG) model was constructed to reduce the number of

593 particles so that computational simulations of hundreds of nanoseconds were

594 feasible. For CG simulations, we used the Martini 2.0 force field, where four

595 heavy (non-hydrogen) atoms were mapped onto a single bead, excluding

596 residues with aromatic side chains. The lipids were modelled as in Gc et al. ${ }^{28}$ and

597 Van Der Spoel et al. ${ }^{51}$. The system was solvated using standard Martini water

598 beads and was neutralized in $0.15 \mathrm{M} \mathrm{NaCl}$. The total number of particles for the

599 CG system was 52827. 
The coarse-grained molecular dynamics simulation was performed using

601 Gromacs 5.1.1 $1^{51,52}$. The simulation was performed with 15 fs time steps. The

602 Lennard-Jones and Coulomb potential had cutoffs at $11 \AA$. In order to keep track

603 of particles, a Verlet neighbor scheme was used and Coulomb interactions were

604 treated using a reaction field. The LINCS algorithm was used to control stiff

605 bonds. The pressure was maintained at 1 bar using a Berendsen barostat for

606 equilibration runs and a Parrinello-Rahman barostat during production runs.

607 Pressure coupling between the protein and membrane was semi-isotropic with a

608 compressibility of $3 \times 10^{-4} \mathrm{bar}^{-1}$ and the temperature was maintained at $303 \mathrm{~K}$.

609 Temperature coupling was done using a velocity rescale algorithm.

\section{Acknowledgments:}

612 This research was supported by NIH AI081077 (R.V.S) and1U19AI117905,

613 R01GM020501, R01NS070899, and R01GM121964 (S.L.). K.A.J would like to

614 thank the NDIIF and Dr. Sergi Rouvimov and Dr. Tatyana Orlova for helpful

615 discussions in TEM and assistance in SEM, respectively. The confocal and

616 multiphoton imaging studies were supported by the Indiana University School of

617 Medicine-South Bend Imaging and Flow Cytometry Core.

\section{References:}

6201 Cantoni, D. \& Rossman, J. S. Ebolaviruses: New roles for old proteins. PLoS

$621 \quad$ Negl Trop Dis 12, e0006349, doi:10.1371/journal.pntd.0006349 (2018).

6222 Emanuel, J., Marzi, A. \& Feldmann, H. Filoviruses: Ecology, Molecular Biology,

623 and Evolution. Adv Virus Res 100, 189-221, doi:10.1016/bs.aivir.2017.12.002

624 (2018). 


\begin{tabular}{|c|c|c|}
\hline $\begin{array}{l}625 \\
626\end{array}$ & 3 & $\begin{array}{l}\text { Suder, E., Furuyama, W., Feldmann, H., Marzi, A. \& de Wit, E. The vesicular } \\
\text { stomatitis virus-based Ebola virus vaccine: from concept to clinical trials. }\end{array}$ \\
\hline 27 & & Hum Vaccin Immunother, 1-18, doi:10.1080/21645515.2018.1473698 \\
\hline 28 & & (2018). \\
\hline 29 & 4 & Gire, S. K. et al. Genomic surveillance elucidates Ebola virus origin and \\
\hline & & transmission during the 2014 outbreak. Science 345, 1369 \\
\hline 1 & & doi:10.1126/science.1259657 (2014). \\
\hline 2 & 5 & Kugelman, J. R. et al. Evaluation of the potential impact of Ebola virus \\
\hline 3 & & genomic drift on the efficacy of sequence-based candidate therapeutics. MBio \\
\hline 34 & & 6, doi:10.1128/mBio.02227-14 (2015). \\
\hline 35 & 6 & Park, D. J. et al. Ebola Virus Epidemiology, Transmission, and Evolution \\
\hline & & during Seven Months in Sierra Leone. Cell 161, 1516-1526, \\
\hline 37 & & doi:10.1016/j.cell.2015.06.007 (2015). \\
\hline 38 & 7 & Miller, C. R. et al. Initiating a watch list for Ebola virus antibody escape \\
\hline 39 & & mutations. PeerJ 4, e1674, doi:10.7717/peerj.1674 (2016). \\
\hline 0 & 8 & Han, Z. et al. Small-molecule probes targeting the viral PPxY-host Nedd4 \\
\hline 41 & & interface block egress of a broad range of RNA viruses. J Virol 88, 7294-7306, \\
\hline 642 & & doi:10.1128/JVI.00591-14 (2014). \\
\hline 43 & 9 & Stahelin, R. V. Could the Ebola virus matrix protein VP40 be a drug target? \\
\hline 44 & & Expert Opin Ther Targets 18, 115-120, doi:10.1517/14728222.2014.863877 \\
\hline 45 & & $(2014)$. \\
\hline 546 & 10 & Madara, J. J., Han, Z., Ruthel, G., Freedman, B. D. \& Harty, R. N. The \\
\hline 47 & & multifunctional Ebola virus VP40 matrix protein is a promising therapeutic \\
\hline 648 & & target. Future Virol 10, 537-546, doi:10.2217/fvl.15.6 (2015). \\
\hline 649 & 11 & Harty, R. N. No exit: targeting the budding process to inhibit filovirus \\
\hline 650 & & replication. Antiviral Res 81, 189-197, doi:10.1016/j.antiviral.2008.12.003 \\
\hline 651 & & $(2009)$. \\
\hline 652 & 12 & Jasenosky, L. D. \& Kawaoka, Y. Filovirus budding. Virus Res 106, 181-188, \\
\hline 54 & 13 & Jasenosky I D Neumann G Lukashevich I \& Kav \\
\hline 65 & & VP40-induced particle formation and association with the lipid bilayer.J Virol \\
\hline 656 & & 75, 5205-5214, doi:10.1128/JVI.75.11.5205-5214.2001 (2001). \\
\hline 657 & 14 & Noda, T. et al. Assembly and budding of Ebolavirus. PLoS Pathog 2 \\
\hline 65 & & doi:10.1371/journal.ppat.0020099 (2006). \\
\hline 659 & 15 & Noda, T. et al. Ebola virus VP40 drives the formation of virus-like filamentous \\
\hline 660 & & particles along with GP. J Virol 76, 4855-4865 (2002). \\
\hline 661 & 16 & Stahelin, R. V. Membrane binding and bending in Ebola VP40 assembly and \\
\hline 662 & & egress. Front Microbiol 5, 300, doi:10.3389/fmicb.2014.00300 (2014). \\
\hline 663 & 17 & Dessen, A., Volchkov, V., Dolnik, O., Klenk, H. D. \& Weissenhorn, W. Crystal \\
\hline 664 & & structure of the matrix protein VP40 from Ebola virus. EMBO J 19, 4228- \\
\hline 665 & & 4236, doi:10.1093/emboj/19.16.4228 (2000). \\
\hline 666 & 18 & Bornholdt, Z. A. et al. Structural rearrangement of ebola virus VP40 begets \\
\hline 667 & & ultiple functions in the virus life cycle. Cell 154, 763-774, \\
\hline & & 15 (2013). \\
\hline
\end{tabular}


66919 Nanbo, A., Watanabe, S., Halfmann, P. \& Kawaoka, Y. The spatio-temporal distribution dynamics of Ebola virus proteins and RNA in infected cells. $S c i$

671

672 Rep 3, 1206, doi:10.1038/srep01206 (2013).

673

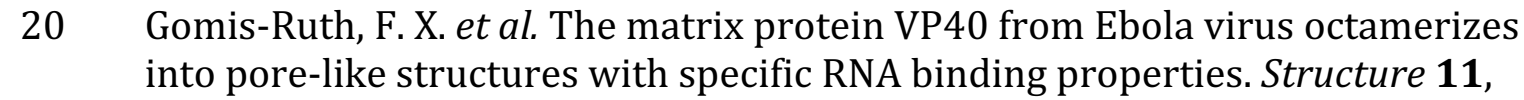

674

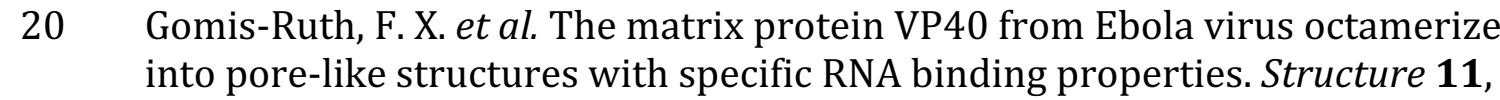
423-433 (2003).

675

21 Adu-Gyamfi, E. et al. Host Cell Plasma Membrane Phosphatidylserine

676

677 Regulates the Assembly and Budding of Ebola Virus. J Virol 89, 9440-9453, doi:10.1128/JVI.01087-15 (2015).

678

679

22 Del Vecchio, K. et al. A cationic, C-terminal patch and structural

680 rearrangements in Ebola virus matrix VP40 protein control its interactions

681 with phosphatidylserine. J Biol Chem 293, 3335-3349,

682 doi:10.1074/jbc.M117.816280 (2018).

683

684

685

686

687

688

689

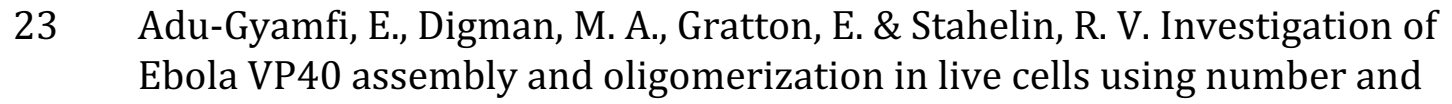
brightness analysis. Biophys J 102, 2517-2525, doi:10.1016/j.bpj.2012.04.022 (2012).

24 Adu-Gyamfi, E. et al. A loop region in the N-terminal domain of Ebola virus VP40 is important in viral assembly, budding, and egress. Viruses 6, 38373854, doi:10.3390/v6103837 (2014).

690

691

692

693

694

695

696

697

25 Adu-Gyamfi, E. et al. The Ebola virus matrix protein penetrates into the plasma membrane: a key step in viral protein 40 (VP40) oligomerization and viral egress. J Biol Chem 288, 5779-5789, doi:10.1074/jbc.M112.443960 (2013).

26 Johnson, K. A., Taghon, G. J., Scott, J. L. \& Stahelin, R. V. The Ebola Virus matrix protein, VP40, requires phosphatidylinositol 4,5-bisphosphate (PI(4,5)P2) for extensive oligomerization at the plasma membrane and viral egress. Sci Rep 6, 19125, doi:10.1038/srep19125 (2016).

698

699

700

27 Gc, J. B. et al. Interdomain salt-bridges in the Ebola virus protein VP40 and their role in domain association and plasma membrane localization. Protein Sci 25, 1648-1658, doi:10.1002/pro.2969 (2016).

701

28 Gc, J. B., Gerstman, B. S., Stahelin, R. V. \& Chapagain, P. P. The Ebola virus protein VP40 hexamer enhances the clustering of PI(4,5)P2 lipids in the

702

703

704

705

706

707 plasma membrane. Phys Chem Chem Phys 18, 28409-28417, doi:10.1039/c6cp03776c (2016).

29 Stahelin, R. V., Scott, J. L. \& Frick, C. T. Cellular and molecular interactions of phosphoinositides and peripheral proteins. Chem Phys Lipids 182, 3-18, doi:10.1016/j.chemphyslip.2014.02.002 (2014).

708

709

710

711 Julkowska, M. M., Rankenberg, J. M. \& Testerink, C. Liposome-binding assays to assess specificity and affinity of phospholipid-protein interactions.

Methods Mol Biol 1009, 261-271, doi:10.1007/978-1-62703-401-2_24 (2013).

31 Johnson, K. A., Taghon, G. J. F., Scott, J. L. \& Stahelin, R. V. The Ebola Virus matrix protein, VP40, requires phosphatidylinositol 4,5-bisphosphate (PI(4,5)P2) for extensive oligomerization at the plasma membrane and viral 
724

725

726

727

728

729

730

731

732

733

734

735

736

737

738

739

740

741

742

743

744

745

746

747

748

749

750

751

752

753

754

755

756

757

758

759
32 Adu-Gyamfi, E. et al. Host Cell Plasma Membrane Phosphatidylserine Regulates the Assembly and Budding of the Ebola Virus. Journal of virology, doi:10.1128/JVI.01087-15 (2015).

33 Stahelin, R. V. Monitoring peripheral protein oligomerization on biological membranes. Methods Cell Biol 117, 359-371, doi:10.1016/B978-0-12408143-7.00019-0 (2013).

34 Santos Mde, S., Naal, R. M., Baird, B. \& Holowka, D. Inhibitors of PI(4,5)P2 synthesis reveal dynamic regulation of IgE receptor signaling by phosphoinositides in RBL mast cells. Mol Pharmacol 83, 793-804, doi:10.1124/mol.112.082834 (2013).

35 Day, A. J. et al. Human metabolism of dietary flavonoids: identification of plasma metabolites of quercetin. Free Radic Res 35, 941-952 (2001).

36 Day, A. J. \& Williamson, G. Human metabolism of dietary quercetin glycosides. Basic Life Sci 66, 415-434 (1999).

37 Harnly, J. M. et al. Flavonoid content of U.S. fruits, vegetables, and nuts. J Agric Food Chem 54, 9966-9977, doi:10.1021/jf061478a (2006).

38 Hertog, M. G. \& Hollman, P. C. Potential health effects of the dietary flavonol quercetin. Eur J Clin Nutr 50, 63-71 (1996).

39 Hollman, P. C., Hertog, M. G. \& Katan, M. B. Role of dietary flavonoids in protection against cancer and coronary heart disease. Biochem Soc Trans 24, 785-789 (1996).

40 Y. Liu, K. S., F. Yang, L. Wituckia and K. M. Shokat. A Molecular Gate which Controls Unnatural ATP Analogue Recognition by the Tyrosine Kinase V-Src. Bioorganic \& Medicinal Chemistry 6, 1219-1226 (1998).

41 Maria Teresa Gatto, S. F., Eleonora Grippa, Gabriela Mazzanti, Lucia Battinelli, Giovanni Nicolosi, Daniela Lambusta and Luciano Saso. Antimicrobial and Anti-Lipase Activity of Quercetin and Its C2-C16 3-O-Acyl-Esters. Bioorganic \& Medicinal Chemistry 10, 269-272 (2002).

42 David R. Ferry, A. S., Joy Malkhandi, David W. Fyfe, Philippa G. deTakats, David Anderson, Jim Baker, and David J. Kerr. Phase I Clinical Trial of the Flavonoid Quercetin: Pharmacokinetics and Evidence for in Vivo Tyrosine Kinase Inhibition. Clinical Cancer Research 2, 659-668 (1996).

43 Wijesinghe, K. J. et al. Detection of lipid-induced structural changes of the Marburg virus matrix protein VP40 using hydrogen/deuterium exchangemass spectrometry. J Biol Chem 292, 6108-6122, doi:10.1074/jbc.M116.758300 (2017).

44 Wijesinghe, K. J. \& Stahelin, R. V. Investigation of the Lipid Binding Properties of the Marburg Virus Matrix Protein VP40. J Virol 90, 3074-3085, doi:10.1128/JVI.02607-15 (2015).

45 GC, J. B. et al. Interdomain salt-bridges in the Ebola virus protein VP40 and their role in domain association and plasma membrane localization. Protein science : a publication of the Protein Society, doi:10.1002/pro.2969 (2016).

46 Barlow, C. A., Laishram, R. S. \& Anderson, R. A. Nuclear phosphoinositides: a signaling enigma wrapped in a compartmental conundrum. Trends in cell biology 20, 25-35, doi:10.1016/j.tcb.2009.09.009 (2010). 
76047 Digman, M. A., Dalal, R., Horwitz, A. F. \& Gratton, E. Mapping the number of

761

762 molecules and brightness in the laser scanning microscope. Biophysical journal 94, 2320-2332, doi:10.1529/biophysj.107.114645 (2008).

48 Gc, J. B., Gerstman, B. S. \& Chapagain, P. P. Membrane association and localization dynamics of the Ebola virus matrix protein VP40. Biochim

765 Biophys Acta 1859, 2012-2020, doi:10.1016/j.bbamem.2017.07.007 (2017).

766

49 Jo, S., Kim, T., Iyer, V. G. \& Im, W. CHARMM-GUI: a web-based graphical user

767

768 interface for CHARMM. Journal of computational chemistry 29, 1859-1865,

$76950 \quad$ Phillips, J. C. et al. Scalable molecular dynamics with NAMD. Journal of

770

771 computational chemistry 26, 1781-1802, doi:10.1002/jcc.20289 (2005).

772

51 Van Der Spoel, D. et al. GROMACS: fast, flexible, and free. J Comput Chem 26,

773

774

775

776

52 Abraham, M. J. et al. GROMACS: High performance molecular simulations through multi-level parallelism from laptops to supercomputers. SoftwareX 1-2, 19-25, doi:http://dx.doi.org/10.1016/j.softx.2015.06.001 (2015). 